\title{
Approximation on partially ordered sets of regular grids
}

\author{
P.W. Hemker ${ }^{\mathrm{a}, *}$, C. Pflaum ${ }^{\mathrm{b}, 1}$ \\ a Centrum voor Wiskunde en Informatica, Kruislaan 413, 1098 SJ Amsterdam, The Netherlands \\ ${ }^{\mathrm{b}}$ Institut für Angewandte Mathematik und Statistik, Am Hubland, 97074 Würzburg, Germany
}

\begin{abstract}
In this paper we analyze the approximation of functions on partially ordered sequences of regular grids. We start with the formulation of minimal requirements for useful grid transfer operators. We introduce the notions of nested and of commutative transfer operators. We define mutual coherence for representations on grids that are not related by coarsening or refining. We show necessary and sufficient conditions for mutual coherence and we show how a hierarchical decomposition is generated by a set of commutative transfer operators. The usual piecewise constant and piecewise $d$-linear approximations are identified as special instances of tensor product type.

In the second part of the paper we derive error estimates for approximation in these spaces, in different norms on general $d$-dimensional dyadic sequences of regular and sparse grids. Some of these results have been published before, e.g., in doctoral theses by Bungartz and Pflaum. Here, the results are presented in a unified framework and the proofs are much simplified. We pay special attention to a convenient notation. $\odot 1997$ Published by Elsevier Science B.V.
\end{abstract}

\section{Introduction and notation}

\subsection{Introduction}

Recently, in the research on multigrid methods for problems in three dimensions more and more attention is paid to semi-coarsening $[5,12,14,17]$ and sparse grid approaches $[2,6-8,13,16]$. This can be understood if we notice that the classical multigrid approach, where a linear sequence of nested grids is used for the approximation on different grids, requires very strong relaxation techniques. The selection of a suitable relaxation is difficult because of the large number of possible choices, each with their particular advantages and disadvantages.

\footnotetext{
${ }^{*}$ Corresponding author. E-mail: p.w.hemker@cwi.nl.

'E-mail: pflaum@mathematik.uni-wuerzburg.de. 
In semi-coarsening, where more coarser grids are introduced, each coarsened in a single direction, the role of the smoothing procedure is reduced, and simpler relaxation procedures can be applied [10]. This makes it attractive to study partially ordered sets of grids, rather than sequentially ordered ones.

Another difficulty, that particularly arises when regular grids are used for the approximation of functions, is the curse of three dimensions: the number of cells increases cubically with each refinement in all directions. This results in enormous amounts of degrees of freedom in the approximation, and in very large systems of algebraic equations to solve. This difficulty can be removed to a large extent by adaptive refinement, i.e., by adding only those degrees of freedom that contribute significantly to the improvement of the accuracy. Of course, what each additional degree of freedom adds to the higher accuracy depends on the choice of basis-functions that span the approximating function space. If, on a regular rectangular grid, a hierarchical basis is chosen, for a sufficiently smooth function the degrees of freedom associated with a "sparse grid" are the optimal choice. The sparse grid can be seen as a combination of regular grids, each with a different cell aspect ratio. In this way semi-coarsening multigrid and sparse grid approximations are much related and make an interesting match.

It appears that the relations between the approximations on the different grids in the partially ordered set are not always clear [4], and that the requirements for the prolongations and restrictions between the approximations on the different grids are often chosen in an ad hoc way. In the present paper we study the approximation of functions on partially ordered sets of regular grids (on a grids of grids). In particular we are interested in the minimal requirements that are needed to introduce the necessary grid transfer operators. Analyzing these requirements results naturally in the introduction of a hierarchical decomposition of the approximation on the grid of grids, and we are able to show how the usual approximations by piecewise constant and piecewise linear basis functions appear as a special case of tensor product form. In the next sections we concentrate on the piecewise constant and piecewise linear approximations. We define their construction in a systematic way and we derive error estimates for the approximations in different norms.

So, the purpose of this paper is twofold: (1) we show what are the essential requirements for prolongations and restrictions to be able to prove the useful properties that are used in computational schemes, such as mutual coherence [4], and (2) we show the approximation properties of piecewise constant and multilinear approximations on anisotropic and sparse grids. In the first part (Section 2) we see that the requirement of nested transfer operators is useful to define restrictions between different grids and to define mutual coherence. Furthermore, we see that we need the stronger requirement of commutativity in order to construct a pre-wavelet decomposition (28). Of course, tensor product spaces give the most interesting and useful examples. In the second part of this paper (Section 3) we derive general error estimates for the simplest cases: piecewise constant and piecewise multilinear approximations.

Studying the approximation on a grid of grids, it appeared that a simple and convenient notation was lacking and that the data structures that are used in practice to realize the related algorithms, are rather complicated. Therefore, in the treatment much attention is given to a convenient notation that can be used in general for the description and analysis of algorithms on a grid of grids [11].

Sparse grids yield a way for obtaining approximations with a high accuracy relative to the number of degrees of freedom (unknowns) used. This was first observed by Smoljak [15] for numerical integration and interpolation with trigonometric functions. A different approach of constructing sparse grids is presented in [16]. This approach uses hierarchical basis functions for interpolation with piecewise multilinear functions. Error estimates in different norms and with different assumptions are found 
in $[2,13,16]$. For obtaining optimal estimates, it is necessary to assume that suitable derivatives of the functions are bounded. In case of singularities these assumptions may not hold. Then, optimal estimates can be obtained on adaptive sparse grids, which can be constructed in a natural way with hierarchical basis functions [2].

\subsection{Notation}

Let $\boldsymbol{k} \in \mathbb{Z}^{d}$ be a multi-integer in $d$ dimensions, then $\boldsymbol{k}=\left(k_{1}, k_{2}, \ldots, k_{d}\right)$, with $k_{i} \in \mathbb{Z}$ for $i=1,2, \ldots, d$. We define relational operators between multi-integers by

$$
\boldsymbol{k}<\boldsymbol{m} \Leftrightarrow\left(k_{1}<m_{1} \text { and } k_{2}<m_{2} \text { and } \ldots \text { and } k_{d}<m_{d}\right) \text {, }
$$

and analogously we define $\boldsymbol{k} \leqslant \boldsymbol{m}, \boldsymbol{k}>\boldsymbol{m}, \boldsymbol{k} \geqslant \boldsymbol{m}$ and $\boldsymbol{k}=\boldsymbol{m}$. Further we define

$$
\max (\boldsymbol{m}, \boldsymbol{n})=\left(\max \left(m_{1}, n_{1}\right), \max \left(m_{2}, n_{2}\right), \ldots, \max \left(m_{d}, n_{d}\right)\right),
$$

and $\min (\boldsymbol{m}, \boldsymbol{n})$ similarly. In a few instances we will use these operators with the same meaning for real vectors $\boldsymbol{x}=\left(x_{1}, \ldots, x_{d}\right) \in \mathbb{R}^{d}$.

With $\boldsymbol{n}=\left(n_{1}, \ldots, n_{d}\right) \in \mathbb{Z}^{d}$ we denote $|\boldsymbol{n}|=n_{1}+\cdots+n_{d}$. We also use the notation $\mathbf{0}=$ $(0, \ldots, 0) \in \mathbb{N}^{d} ; 2^{n}=\left(2^{n_{1}}, \ldots, 2^{n_{d}}\right) ; 2^{n} \boldsymbol{x}=\left(2^{n_{1}} x_{1}, \ldots, 2^{n_{d}} x_{d}\right) ; \boldsymbol{n} \circ \boldsymbol{m}=\sum_{i=1, \ldots, d} n_{i} m_{i}$, and $\|\boldsymbol{n}\|=n_{1} \cdots n_{d}$. Further we introduce in $\mathbb{Z}^{d}$ the unit vectors $\boldsymbol{e}_{k}, k=1, \ldots, d$, as follows: $\boldsymbol{e}_{1}=$ $(1,0, \ldots, 0) ; \boldsymbol{e}_{2}=(0,1,0, \ldots, 0) ; \boldsymbol{e}_{d}=(0, \ldots, 0,1)$, and we use $\boldsymbol{e}=(1, \ldots, 1)$. Finally we define $\boldsymbol{E}=\left\{\boldsymbol{e}_{1}, \ldots, \boldsymbol{e}_{d}\right\}$.

Let either $\Omega=\mathbb{R}^{d}$ be the $d$-dimensional Euclidean space, or let $\Omega=(0,1)^{d} \subset \mathbb{R}^{d}$ be the $d$ dimensional open unit cube. With any multi-integer $n \in \mathbb{Z}^{d}$ we associate a function space $V_{n}$, e.g., the space of piecewise constant or piecewise linear (bi-linear, tri-linear, $d$-linear) functions on a uniform grid with mesh size $\boldsymbol{h}=\left(h_{1}, \ldots, h_{d}\right)=\left(2^{-n_{1}}, \ldots, 2^{-n_{d}}\right)$. These grids are uniformly spaced in each of the $d$ coordinate directions, but possibly with a different mesh size in the different directions. The volume of these cells is denoted by $\|\boldsymbol{h}\|=2^{-|\boldsymbol{n}|}$. The functions in $V_{\boldsymbol{n}}$ all are constant or $d$-linear on each dyadic block or cell

$$
\Omega_{n, k}=\left[k_{1} 2^{-n_{1}},\left(k_{1}+1\right) 2^{-n_{1}}\right] \times \cdots \times\left[k_{d} 2^{-n_{d}},\left(k_{d}+1\right) 2^{-n_{d}}\right],
$$

and this family of cells forms the grid

$$
\Omega_{n}=\left\{\Omega_{n, k} \mid \Omega_{n, k} \subset \bar{\Omega}, k \in \mathbb{Z}^{d}\right\} .
$$

The family of cell centers or cell nodes is denoted by

$$
\Omega_{n}^{*}=\left\{z_{n, k} \mid z_{n, k}=(k+e / 2) 2^{-n} ; k \in \mathbb{Z}^{d} ; z_{n, k} \in \Omega\right\} .
$$

Other grids are obtained by considering the cell vertices or vertex nodes of the cells in $\Omega_{n}$ as a grid of points. We denote these grids by $\Omega_{n}^{+}$.

Apparently, all grids are identified by a multi-integer $\boldsymbol{n}$; the number $|\boldsymbol{n}|$ is called the level of the grid $\boldsymbol{n}$. Notice that-different from classical multigrid theory-we make a clear distinction between the grid-identification $\boldsymbol{n}$ and the level number $|\boldsymbol{n}|$.

We also use the following notation for partial derivatives, with $\boldsymbol{n} \in \mathbb{N}_{0}^{d}$,

$$
D^{n}=D^{n_{1}, \ldots, n_{d}}=\left(\frac{\partial}{\partial x_{1}}\right)^{n_{1}} \cdots\left(\frac{\partial}{\partial x_{d}}\right)^{n_{d}}
$$


For the Banach spaces of continuously differentiable functions we use, with $n \in \mathbb{N}_{0}^{d}$, the notation $C^{n}(\Omega)$ for the space of functions with finite norm

$$
\|u\|_{C^{n}}=\max _{0 \leqslant m \leqslant n} \max _{x \in \Omega}\left|D^{m} u(\boldsymbol{x})\right| \text {. }
$$

For $l \in \mathbb{N}_{0}$, we introduce the notation $\mathcal{C}^{n, l}(\Omega)=\bigcap_{|m|=l} C^{n+m}(\Omega)$. This is a generalization which combines $C^{n}(\Omega)=\mathcal{C}^{n, 0}(\Omega)$ with the usual space of $l$ times continuously differentiable functions $C^{l}(\Omega)=\mathcal{C}^{0, l}(\Omega)$. With a $C_{0}^{n}(\Omega)$ and $\mathcal{C}_{0}^{n, l}(\Omega)$ we denote the corresponding subspaces with homogeneous boundary conditions.

For the Banach spaces of integrable functions, $1 \leqslant p \leqslant \infty$, we, similarly, use the notation $W_{p}^{n}(\Omega)$ for the space of functions with finite norm

$$
\|u\|_{W_{p}^{n}}=\left(\sum_{0 \leqslant m \leqslant n}|u|_{W_{p}^{m}}^{p}\right)^{1 / p}
$$

Further we use the semi-norm $|u|_{W_{p}^{n}}$ or, with $0 \leqslant k \leqslant d$, the norm $\|u\|_{W_{p}^{n, k}}$, defined by

$$
|u|_{W_{p}^{n}}=\left(\int_{x \in \Omega}\left|D^{n} u(x)\right|^{p}\right)^{1 / p} \text { and }\|u\|_{W_{p}^{n, k}}=\left(\sum_{0 \leqslant m \leqslant e,|m|=k}\left\|D^{n+m} u\right\|_{p}^{p}\right)^{1 / p} .
$$

For $l \in \mathbb{N}_{0}$, we write $\mathcal{W}_{p}^{n, l}(\Omega)=\bigcap_{|m|=l} W_{p}^{n+m}(\Omega)$ and we obtain the Sobolev space $W_{p}^{l}(\Omega)=$ $\mathcal{W}_{p}^{\mathbf{0}, l}(\Omega)$. Again, with a $W_{p, 0}^{l}(\Omega)$ and $\mathcal{W}_{p, 0}^{n, l}(\Omega)$ we denote the corresponding subspaces with homogeneous boundary conditions. For $p=\infty$ we use the standard modifications, and for $W_{2}$ we also write $H$. Thus, for the Hilbert spaces of square integrable functions we use the notation $H^{n}(\Omega)=W_{2}^{n}(\Omega)$, and for the semi-norm and norm $|u|_{H^{n}}=|u|_{W_{2}^{n}}$ and $\|u\|_{H^{n}}=\|u\|_{W_{2}^{n}}$. For $l \in \mathbb{N}_{0}$, we write $\mathcal{H}^{n, l}(\Omega)=\bigcap_{|\boldsymbol{m}|=l} H^{\boldsymbol{n}+\boldsymbol{m}}(\Omega)$ and we obtain the usual Sobolev space $H^{l}(\Omega)=\mathcal{H}^{\mathbf{0}, l}(\Omega)$. Again, with a $H_{0}^{l}(\Omega)$ and $\mathcal{H}_{0}^{n, l}(\Omega)$ we denote the corresponding subspaces with homogeneous boundary conditions.

\section{Space decomposition}

In this section we introduce nested and commutative sets of restrictions and prolongations. We see that the nested property is required to define restrictions between coarser and finer spaces. The property of commutativity is needed to check coherence of approximations on different grids, and, moreover, it allows characterization of approximating spaces by a hierarchical decomposition.

\subsection{Nested restrictions and prolongations}

Let $X$ be a Banach space; e.g., $X=C^{0}(\Omega), X=L_{p}(\Omega)$ or $X=L_{p}^{\text {loc }}(\Omega)$, where $\Omega \subset \mathbb{R}^{d}$. Let $\boldsymbol{k} \in \mathbb{Z}^{d}$ and let

$$
R_{k}: X \rightarrow V_{k}
$$


be a restriction, i.e., a linear surjection. Possibly $V_{k} \subset X$, but this is not necessary. We notice that for any such $R_{k}$, because of the surjection, there exists the right-inverse or reconstruction

$$
P_{k}: V_{k} \rightarrow X,
$$

such that

$$
R_{k} P_{k}=I_{k}
$$

is the identity operator on $V_{\boldsymbol{k}}$. We notice that $P_{\boldsymbol{k}}$ is an injection (and hence a prolongation) and $\operatorname{Ran}\left(P_{\boldsymbol{k}}\right) \subset X$, but $P_{\boldsymbol{k}}$ is not uniquely determined by a given $R_{\boldsymbol{k}}$. In this section we study properties of such sets of transfer operators $\left\{R_{\boldsymbol{k}}\right\}_{\boldsymbol{k} \in \mathbb{Z}^{d}}$ and $\left\{P_{\boldsymbol{k}}\right\}_{\boldsymbol{k} \in \mathbb{Z}^{d}}$.

It is a consequence of (3) that

$$
\Pi_{k}=P_{k} R_{k}
$$

is a projection

$$
\Pi_{\boldsymbol{k}}: X \rightarrow \operatorname{Ran}\left(\Pi_{\boldsymbol{k}}\right)=\operatorname{Ran}\left(P_{k}\right) \subset X
$$

as is

$$
I-\Pi_{k}: X \rightarrow \operatorname{Ker}\left(\Pi_{k}\right)=\operatorname{Ker}\left(R_{k}\right) \subset X
$$

and we observe that $X$ can be written as a direct $\operatorname{sum} X=\operatorname{Ran}\left(P_{k}\right) \oplus \operatorname{Ker}\left(R_{k}\right)$.

Definition 2.1. A set $\left\{R_{\boldsymbol{k}}\right\}_{\boldsymbol{k} \in \mathbb{Z}^{d}}$ is called a nested set of restrictions (or NSR) iff

$$
\boldsymbol{k} \geqslant \boldsymbol{m} \Rightarrow \operatorname{Ker}\left(R_{\boldsymbol{k}}\right) \subset \operatorname{Ker}\left(R_{\boldsymbol{m}}\right) \text {. }
$$

A set $\left\{P_{\boldsymbol{k}}\right\}_{\boldsymbol{k} \in \mathbb{Z}^{d}}$ is called a nested set of prolongations (or NSP) iff

$$
\boldsymbol{k} \geqslant \boldsymbol{m} \Rightarrow \operatorname{Ran}\left(P_{\boldsymbol{k}}\right) \supset \operatorname{Ran}\left(P_{\boldsymbol{m}}\right)
$$

It is obvious that for an NSR $\left\{R_{k}\right\}$ a set of corresponding reconstructions is not necessarily an NSP. On the other hand, given an NSP, the corresponding set of restrictions is not necessarily an NSR. However, in some cases both the restrictions and their reconstructions may form nested sets. Then we say that the transfer operators are nested and $\left\{V_{k}\right\}$ forms a nested set of representations of functions in $X$.

Theorem 2.2. Let $\left\{R_{\boldsymbol{k}}\right\}_{\boldsymbol{k} \in \mathbb{Z}^{d}}$ be a nested set of restrictions, and $\left\{P_{\boldsymbol{k}}\right\}_{\boldsymbol{k} \in \mathbb{Z}^{d}}$ a set of corresponding reconstructions, then

$$
\forall \boldsymbol{n} \geqslant \boldsymbol{m} \exists ! R_{m n}: V_{n} \rightarrow V_{m}
$$

with the properties:

$$
\begin{aligned}
& \text { (1) } R_{m n} \text { is a restriction; } \\
& \text { (2) } R_{m n} R_{n}=R_{m} ; \\
& \text { (3) } R_{m n}=R_{m} P_{n} \quad \text { (independent of the choice of } P_{n} \text { !). }
\end{aligned}
$$

Proof. (i) Define $R_{m n}^{1}=R_{m} P_{n}^{1}$ and $R_{m n}^{2}=R_{m} P_{n}^{2}$. Then we know that $R_{n} P_{n}^{1}=I_{n}=R_{n} P_{n}^{2}$ and hence $\forall v_{n} \in V_{n} R_{n}\left(P_{n}^{1}-P_{n}^{2}\right) v_{n}=0$. Because $\left\{R_{k}\right\}$ is an NSR and $\boldsymbol{m} \leqslant \boldsymbol{n}$ it follows that 
$R_{m}\left(P_{n}^{1}-P_{n}^{2}\right) v_{n}=0$ and hence $R_{m n}^{1}=R_{m n}^{2}$. So that there exists a unique $R_{m n}$. This means that we can write $R_{m n}=R_{m} P_{n}$, and $R_{m n}$ is independent of the choice of $P_{n}$.

(ii) $R_{m n} R_{n}=R_{m} P_{n} R_{n}=R_{m} \Pi_{n}=R_{m}$ on $\operatorname{Ran}\left(P_{n}\right)$. Now, because $X=\operatorname{Ran}\left(P_{n}\right) \oplus \operatorname{Ker}\left(R_{n}\right)$ we may write $\forall v \in X: v=v_{p}+v_{n}$ so that

$$
R_{m n} R_{n} v=R_{m n} R_{n} v_{p}+R_{m n} R_{n} v_{n}=R_{m} v_{p}+0=R_{m} v_{p}
$$

Further, because of $\operatorname{Ker}\left(R_{n}\right) \subset \operatorname{Ker}\left(R_{m}\right)$ we see $R_{m} v=R_{m} v_{p}+R_{m} v_{n}=R_{m} v_{p}+0=R_{m} v_{p}$, so that $R_{m n} R_{n} v=R_{m} v_{p}=R_{m} v \forall v \in X$ and hence $R_{m n} R_{n}=R_{m}$.

(iii) Because $R_{m}$ is a surjection, and by (6), $R_{m n}$ is necessarily a surjection. Of course, $R_{m n}$ is linear (trivial). Therefore $R_{m n}$ is a restriction.

Given an NSR $\left\{R_{m}\right\}_{m \in \mathbb{Z}^{d}}$, and a set of corresponding reconstructions $\left\{P_{\boldsymbol{m}}\right\}_{\boldsymbol{m} \in \mathbb{Z}^{d}}$, we introduce, for $\boldsymbol{m} \leqslant \boldsymbol{n}$,

$$
P_{n m}=R_{n} P_{m}: V_{m} \rightarrow V_{n} .
$$

Notice that there are many possible choices of $P_{m}$ for a give $R_{m}$. Of course, some actual properties of $P_{n m}$ may depend on this choice!

Lemma 2.3. $P_{n m}$ is a right inverse of $R_{n m}$ :

$$
R_{m n} P_{n m}=I_{m}
$$

Proof. $R_{m n} P_{n m}=R_{m} P_{n} R_{n} P_{m}=R_{m} P_{m}-R_{m}\left(I-P_{n} R_{n}\right) P_{m}=I_{m}+0$, because $\operatorname{Ker}\left(R_{n}\right) \subset$ $\operatorname{Ker}\left(R_{m}\right)$.

\section{Corollary 2.4. With $n \geqslant m$ :}

(1) $P_{n m}$ is a prolongation (i.e., a linear injection);

(2) $P_{n m} R_{m n}$ is a projection $V_{n} \rightarrow \operatorname{Ran}\left(P_{n m}\right) \subset V_{n}$;

(3) $I_{n}-P_{n m} R_{m n}$ is a projection $V_{n} \rightarrow \operatorname{Ker}\left(R_{m n}\right) \subset V_{n}$;

(4) $V_{n}=\operatorname{Ran}\left(P_{n m}\right) \oplus \operatorname{Ker}\left(R_{m n}\right)$;

(5) $P_{n m}: V_{m} \rightarrow \operatorname{Ran}\left(P_{n m}\right) \subset V_{n}$ is a bijection.

Lemma 2.5. Let $\left\{R_{k}\right\}_{\boldsymbol{k} \in \mathbb{Z}^{d}}$ be an NSR and let $\boldsymbol{k} \geqslant \boldsymbol{n} \geqslant \boldsymbol{l}$, then with a given set of corresponding reconstructions $\left\{P_{k}\right\}_{k \in \mathbb{Z}^{d}}$ we have

$$
P_{k l}=P_{k n} P_{n l} \text {. }
$$

\section{Proof.}

$$
\begin{aligned}
P_{k n} P_{n l} & =R_{k} P_{n} R_{n} P_{l}=R_{k} P_{l}-R_{k}\left(I-P_{n} R_{n}\right) P_{l}=R_{k} P_{l}-R_{k}\left(I-\Pi_{n}\right) P_{l} \\
& =R_{k} P_{l}-0=R_{k} P_{l},
\end{aligned}
$$

because $\operatorname{Ker}\left(R_{\boldsymbol{k}}\right) \subset \operatorname{Ker}\left(\Pi_{\boldsymbol{n}}\right)$. Hence

$$
P_{k l}=R_{k} P_{l}=P_{k n} P_{n l} \text {. }
$$

Lemma 2.6. Let $\left\{R_{k}\right\}_{\boldsymbol{k} \in \mathbb{Z}^{d}}$ be an NSR with the corresponding $\left\{P_{\boldsymbol{k}}\right\}_{\boldsymbol{k} \in \mathbb{Z}^{d}}$ an NSP, then

$$
\boldsymbol{m} \geqslant l \Rightarrow P_{\boldsymbol{m}} P_{m l}=P_{l} \text {. }
$$


Proof. $P_{m} P_{m l}=P_{m} R_{m} P_{l}=\Pi_{m} P_{l}=P_{l}$. The last equality holding because $\Pi_{m} u=u$ for all $u \in \operatorname{Ran}\left(\Pi_{m}\right)=\operatorname{Ran}\left(P_{m}\right) \supset \operatorname{Ran}\left(P_{l}\right)$.

Lemma 2.7. If $\left\{R_{k}\right\}_{k \in \mathbb{Z}^{d}}$ is an NSR, then

$$
\boldsymbol{m} \geqslant l \Rightarrow \Pi_{l} \Pi_{\boldsymbol{m}}=\Pi_{l} \text {. }
$$

If, in addition, $\left\{P_{\boldsymbol{k}}\right\}_{\boldsymbol{k} \in \mathbb{Z}^{d}}$ is an NSP, then also

$$
\boldsymbol{m} \geqslant l \Rightarrow \Pi_{m} \Pi_{l}=\Pi_{l} \text {. }
$$

Proof. The first equality follows by

$$
\Pi_{l} \Pi_{m}=P_{l} R_{l} P_{m} R_{m}=P_{l} R_{l m} R_{m}=P_{l} R_{l}=\Pi_{l}
$$

and the second equality by

$$
\Pi_{m} \Pi_{l}=P_{m} R_{m} P_{l} R_{l}=P_{m} R_{m} P_{m} P_{m l} R_{l m} R_{m}=P_{m} P_{m l} R_{l m} R_{m}=P_{l} R_{l}=\Pi_{l}
$$

If $\left\{R_{\boldsymbol{k}}\right\}_{\boldsymbol{k} \in \mathbb{Z}^{d}}$ is an NSR, and if $\boldsymbol{m} \leqslant \boldsymbol{n}$, then a bijection exists between $\operatorname{Ran}\left(P_{\boldsymbol{m}}\right)$ and a subset of $\operatorname{Ran}\left(P_{n}\right)$. We denote this relation by $\operatorname{Ran}\left(P_{m}\right) \preceq \operatorname{Ran}\left(P_{n}\right)$. I.e., a function that can be found in $\operatorname{Ran}\left(P_{m}\right)$, can uniquely be associated with a function in $\operatorname{Ran}\left(P_{n}\right)$. This follows because a bijection exists between $\operatorname{Ran}\left(P_{n}\right)$ and $V_{n}$, and between $\operatorname{Ran}\left(P_{m}\right)$ and $V_{m}$; and also a bijection exists between $V_{m}$ and $\operatorname{Ran}\left(P_{n m}\right) \subset V_{n}$.

Hence, given a Banach space $X$ with a nested set of restrictions $\left\{R_{\boldsymbol{k}}\right\}_{\boldsymbol{k} \in \mathbb{Z}^{d}}$, a family of subspaces $\operatorname{Ran}\left(P_{\boldsymbol{n}}\right)$ exists, with a partial ordering corresponding with the partial ordering of $\{\boldsymbol{n}\}$. This means that, although not necessarily $\operatorname{Ran}\left(P_{m}\right) \subset \operatorname{Ran}\left(P_{n}\right)$, a partial ordering exists such that

$$
\boldsymbol{m} \leqslant \boldsymbol{n} \Leftrightarrow \operatorname{Ran}\left(P_{\boldsymbol{m}}\right) \preceq \operatorname{Ran}\left(P_{n}\right) \Leftrightarrow P_{n m} R_{m} \operatorname{Ran}\left(P_{m}\right)=R_{\boldsymbol{n}} \operatorname{Ran}\left(P_{m}\right) \subset R_{n} \operatorname{Ran}\left(P_{n}\right) .
$$

If $\left\{P_{\boldsymbol{k}}\right\}_{\boldsymbol{k} \in \mathbb{Z}^{d}}$ is an NSP, this partial ordering simply reduces to $\operatorname{Ran}\left(P_{\boldsymbol{m}}\right) \subset \operatorname{Ran}\left(P_{\boldsymbol{n}}\right)$. Then, in the case that $V_{\boldsymbol{n}} \subset X$ and we take $P_{\boldsymbol{n}}$ to be the natural injection, this means that $\operatorname{Ran}\left(P_{\boldsymbol{n}}\right)$ can be identified with $V_{\boldsymbol{n}}$ and $\boldsymbol{m} \leqslant \boldsymbol{n} \Leftrightarrow V_{\boldsymbol{m}} \subset V_{\boldsymbol{n}}$ and, thus, we find $\left\{V_{\boldsymbol{n}}\right\}$ to be a partially ordered family of subsets of $X$.

Definition 2.8. Functions $f_{m} \in V_{m}$ and $f_{n} \in V_{n}$ are mutually coherent ${ }^{2}$ iff

$\exists f_{\boldsymbol{k}} \in V_{\boldsymbol{k}} \quad$ with $\boldsymbol{k} \geqslant \boldsymbol{m}, \boldsymbol{k} \geqslant \boldsymbol{n}$,

such that $f_{m}=R_{m k} f_{k}$ and $f_{n}=R_{n k} f_{k}$.

Theorem 2.9. Let $\left\{R_{\boldsymbol{k}}\right\}_{\boldsymbol{k} \in \mathbb{Z}^{d}}$ be an NSR and $\left\{P_{\boldsymbol{k}}\right\}_{\boldsymbol{k} \in \mathbb{Z}^{d}}$ an NSP. Then, if $f_{\boldsymbol{m}}$ and $f_{\boldsymbol{n}}$ are mutually
coherent, we have

$\forall \boldsymbol{l} \in \mathbb{Z}^{d}$ with $\boldsymbol{l} \leqslant \boldsymbol{m}, \boldsymbol{l} \leqslant \boldsymbol{n}$, we have $R_{\boldsymbol{l m}} f_{\boldsymbol{m}}=R_{\boldsymbol{l n}} f_{\boldsymbol{n}}$.

Moreover, under the additional condition that $\Pi_{\min (\boldsymbol{m}, n)}=\Pi_{m} \Pi_{n}$, also the reverse holds: it follows from (9) that $f_{m}$ and $f_{n}$ are mutually coherent.

\footnotetext{
${ }^{2}$ For the practical use of mutual coherence see, e.g., [4].
} 
Proof. $(\Rightarrow)$ First we assume that $f_{m} \in V_{m}$ and $f_{n} \in V_{n}$ are mutually coherent. Then $\exists f_{k} \in V_{k}$ with $k \geqslant m, k \geqslant n$ such that $f_{m}=R_{m k} f_{k}, f_{n}=R_{n k} f_{k}$, and hence

$$
R_{l m} f_{m}=R_{l m} R_{m k} f_{k}=R_{l k} f_{k}=R_{l n} R_{n k} f_{k}=R_{l n} f_{n}
$$

which proves (9).

$(\Leftarrow)$ Now we assume $(9)$. Let $\boldsymbol{k}=\max (\boldsymbol{m}, \boldsymbol{n})$ and take $\boldsymbol{l}=\min (\boldsymbol{m}, \boldsymbol{n})$. We introduce $f_{\boldsymbol{k}}$ by

$$
f_{k}=P_{k m} f_{m}+P_{k n} f_{n}-P_{k l} R_{l m} f_{m}=P_{k m} f_{m}+P_{k n} f_{n}-P_{k l} R_{l n} f_{n}
$$

Then

$$
\begin{aligned}
R_{m k} f_{k} & =R_{m k} P_{k m} f_{m}+R_{m k} P_{k n} f_{n}-R_{m k} P_{k l} R_{l n} f_{n} \\
& =R_{m k} P_{k m} f_{m}+R_{m k} P_{k n}\left(I_{n}-P_{n l} R_{l n}\right) f_{n} .
\end{aligned}
$$

Now, because $\boldsymbol{k} \geqslant \boldsymbol{m}$ and $\boldsymbol{k} \geqslant \boldsymbol{n} \geqslant \boldsymbol{l}$, we know $R_{\boldsymbol{m} \boldsymbol{k}} P_{\boldsymbol{k} \boldsymbol{m}}=I_{\boldsymbol{m}}$ (Lemma 2.3).

The additional condition and Lemma 2.7 show $\Pi_{m} \Pi_{n}=\Pi_{l}=\Pi_{m} \Pi_{l} \Pi_{n}$, so that $\Pi_{m}(I-$ $\left.\Pi_{l}\right) \Pi_{n}=0$. Hence,

$$
\begin{aligned}
R_{m k} P_{k n}\left(I_{n}-P_{n l} R_{l n}\right) & =R_{m} P_{k} R_{k} P_{n}\left(I_{n}-R_{n} P_{l} R_{l} P_{n}\right) \\
& =R_{m} P_{k} R_{k}\left(I-P_{n} R_{n} P_{l} R_{l}\right) P_{n} \\
& =R_{m} \Pi_{m} \Pi_{k}\left(I-\Pi_{n} \Pi_{l}\right) \Pi_{n} P_{n} \\
& =R_{m} \Pi_{m}\left(I-\Pi_{l}\right) \Pi_{n} P_{n}=0
\end{aligned}
$$

Thus, we find $R_{m k} f_{k}=f_{m}+0=f_{m}$. Analogously we prove $R_{n k} f_{k}=f_{n}$.

\subsection{Commutative restrictions and projections}

Definition 2.10. A set $\left\{R_{k}\right\}_{k \in \mathbb{Z}^{d}}$ is called a commutative set of restrictions (or a CSR) iff for all $\boldsymbol{m}, \boldsymbol{n} \in \mathbb{Z}^{d}$

$$
\begin{gathered}
\operatorname{Ker}\left(R_{n}\right) \cap \operatorname{Ker}\left(R_{m}\right) \\
\oplus \\
\operatorname{Ker}\left(R_{\min (\boldsymbol{m}, \boldsymbol{n})}\right)=\operatorname{Ker}\left(R_{n}\right) \cap \operatorname{Ran}\left(P_{\boldsymbol{m}}\right) . \\
\oplus \\
\operatorname{Ran}\left(P_{n}\right) \cap \operatorname{Ker}\left(R_{\boldsymbol{m}}\right)
\end{gathered}
$$

A set $\left\{P_{\boldsymbol{k}}\right\}_{\boldsymbol{k} \in \mathbb{Z}^{d}}$ is called a commutative set of prolongations (or a CSP) iff for all $\boldsymbol{m}, \boldsymbol{n} \in \mathbb{Z}^{d}$

$$
\operatorname{Ran}\left(P_{\min (\boldsymbol{m}, \boldsymbol{n})}\right)=\operatorname{Ran}\left(P_{n}\right) \cap \operatorname{Ran}\left(P_{m}\right) .
$$

If $\left\{R_{k}\right\}_{\boldsymbol{k} \in \mathbb{Z}^{d}}$ is a CSR and $\left\{P_{\boldsymbol{k}}\right\}_{\boldsymbol{k} \in \mathbb{Z}^{d}}$ is a CSP, then we say that we have commutative transfer operators.

It is immediate that each CSR is an NSR and each CSP is an NSP: which simply follows from the equivalence $n \leqslant m \Leftrightarrow n=\min (\boldsymbol{n}, \boldsymbol{m})$. On the other hand not necessarily every NSP is a CSP nor every NSR a CSR. 
In the next theorem we show how the above definition of a CSR and an NSP lead to commutative projection operators indeed. To prove the theorem, we derive the following three lemmas.

Lemma 2.11. If (12) holds, then $\left\{R_{\boldsymbol{k}}\right\}_{k \in \mathbb{Z}^{d}}$ is a CSR.

Proof. Let $\boldsymbol{m}, \boldsymbol{n} \in \mathbb{Z}^{d}$ be arbitrary, and let $\boldsymbol{l}=\min (\boldsymbol{m}, \boldsymbol{n})$, then we know by assumption $\Pi_{\boldsymbol{m}} \Pi_{\boldsymbol{n}}=$ $\Pi_{l}=\Pi_{n} \Pi_{m}$. We define

$$
M=\operatorname{Span}\left(\left(\operatorname{Ker}\left(R_{n}\right) \cap \operatorname{Ker}\left(R_{m}\right)\right),\left(\operatorname{Ker}\left(R_{n}\right) \cap \operatorname{Ran}\left(P_{m}\right)\right),\left(\operatorname{Ran}\left(P_{n}\right) \cap \operatorname{Ker}\left(R_{\boldsymbol{m}}\right)\right)\right) .
$$

To prove the lemma we show:

(i) $M \subset \operatorname{Ker}\left(\Pi_{l}\right)$,

(ii) $\operatorname{Ker}\left(\Pi_{\boldsymbol{l}}\right) \subset M$,

(iii) $M=\left(\operatorname{Ker}\left(R_{n}\right) \cap \operatorname{Ker}\left(R_{m}\right)\right) \oplus\left(\operatorname{Ker}\left(R_{n}\right) \cap \operatorname{Ran}\left(P_{m}\right)\right) \oplus\left(\operatorname{Ran}\left(P_{n}\right) \cap \operatorname{Ker}\left(R_{m}\right)\right)$.

To prove (i), let $x \in M$, then $x=x_{m}+x_{n}+x_{l}$ with $x_{n} \in \operatorname{Ker}\left(\Pi_{\boldsymbol{n}}\right) \cap \operatorname{Ran}\left(\Pi_{m}\right), x_{m} \in$ $\operatorname{Ker}\left(\Pi_{m}\right) \cap \operatorname{Ran}\left(\Pi_{n}\right), x_{l} \in \operatorname{Ker}\left(\Pi_{n}\right) \cap \operatorname{Ker}\left(\Pi_{m}\right)$. Then

$$
\Pi_{l} x=\Pi_{\boldsymbol{n}} \Pi_{\boldsymbol{m}} x_{m}+\Pi_{m} \Pi_{\boldsymbol{n}} x_{n}+\Pi_{m} \Pi_{\boldsymbol{n}} x_{l}=\Pi_{\boldsymbol{n}} 0+\Pi_{\boldsymbol{m}} 0+\Pi_{\boldsymbol{m}} 0=0 .
$$

So that $x \in \operatorname{Ker}\left(\Pi_{l}\right)$.

To prove (ii), let $x \in \operatorname{Ker}\left(\Pi_{l}\right)$ be arbitrary and define $x_{m}:=\Pi_{m}\left(I-\Pi_{n}\right) x=\Pi_{m} x$ and $x_{n}:=$ $\Pi_{\boldsymbol{n}}\left(I-\Pi_{\boldsymbol{m}}\right) x=\Pi_{\boldsymbol{n}} x$ and

$$
x_{0}:=\left(I-\Pi_{m}-\Pi_{n}+\Pi_{n} \Pi_{m}\right) x=\left(I-\Pi_{m}-\Pi_{n}\right) x
$$

Then a simple calculation shows: $x_{0} \in \operatorname{Ker}\left(R_{n}\right) \cap \operatorname{Ker}\left(R_{m}\right)$ and $x_{m} \in \operatorname{Ker}\left(R_{n}\right) \cap \operatorname{Ran}\left(P_{m}\right)$ and $x_{n} \in \operatorname{Ran}\left(P_{\boldsymbol{n}}\right) \cap \operatorname{Ker}\left(R_{\boldsymbol{m}}\right)$ and $x_{0}+x_{m}+x_{n}=x$.

To prove (iii), we show that $M$ is a direct sum of the three spanning spaces. For this we have to prove: if $x_{0} \in \operatorname{Ker}\left(R_{n}\right) \cap \operatorname{Ker}\left(R_{m}\right)$ and $x_{m} \in \operatorname{Ker}\left(R_{n}\right) \cap \operatorname{Ran}\left(P_{m}\right)$ and $x_{n} \in \operatorname{Ran}\left(P_{n}\right) \cap \operatorname{Ker}\left(R_{m}\right)$ and $x_{0}+x_{m}+x_{n}=0$, then $x_{0}=x_{m}=x_{n}=0$. This is seen by $0=\Pi_{m}\left(I-\Pi_{n}\right)\left(x_{0}+x_{m}+x_{n}\right)=x_{m}$ and $0=\Pi_{n}\left(I-\Pi_{m}\right)\left(x_{0}+x_{m}+x_{n}\right)=x_{n}$. This implies $x_{0}=0$.

Lemma 2.12. If (12) holds, then $\left\{P_{\boldsymbol{k}}\right\}_{\boldsymbol{k} \in \mathbb{Z}^{d}}$ is an NSP.

Proof. Let $\boldsymbol{n}<\boldsymbol{m}$ then (12) implies $\Pi_{\boldsymbol{m}} \Pi_{\boldsymbol{n}}=\Pi_{\boldsymbol{n}}$, and hence, for all $x \in X$ we have $\Pi_{m} \Pi_{n} x=$ $\Pi_{n} x$. It follows that for all $x \in X$ holds $\Pi_{n} x \in \operatorname{Ran}\left(\Pi_{m}\right)$. Therefore $\operatorname{Ran}\left(\Pi_{n}\right) \subset \operatorname{Ran}\left(\Pi_{m}\right)$ for all $\boldsymbol{m}, \boldsymbol{n} \in \mathbb{Z}^{d}$ with $\boldsymbol{n}<\boldsymbol{m}$. Hence $\left\{P_{\boldsymbol{k}}\right\}_{\boldsymbol{k} \in \mathbb{Z}^{d}}$ is a NSP.

Lemma 2.13. If $\left\{R_{\boldsymbol{k}}\right\}_{\boldsymbol{k} \in \mathbb{Z}^{d}}$ is a CSR and $\left\{P_{\boldsymbol{k}}\right\}_{\boldsymbol{k} \in \mathbb{Z}^{d}}$ is an NSP then (12) holds.

Proof. Let $\boldsymbol{l}=\min (\boldsymbol{m}, \boldsymbol{n})$ and let $x \in X$ be arbitrary. We know that

$$
\begin{aligned}
X & =\operatorname{Ran}\left(P_{\boldsymbol{l}}\right) \oplus \operatorname{Ker}\left(R_{l}\right) \\
& =\operatorname{Ran}\left(P_{\boldsymbol{l}}\right) \oplus\left(\operatorname{Ker}\left(R_{\boldsymbol{n}}\right) \cap \operatorname{Ker}\left(R_{\boldsymbol{m}}\right)\right) \oplus\left(\operatorname{Ran}\left(P_{\boldsymbol{n}}\right) \cap \operatorname{Ker}\left(R_{\boldsymbol{m}}\right)\right) \oplus\left(\operatorname{Ker}\left(R_{\boldsymbol{n}}\right) \cap \operatorname{Ran}\left(P_{\boldsymbol{m}}\right)\right)
\end{aligned}
$$

because $\left\{R_{\boldsymbol{k}}\right\}$ is a CSR. Hence, we may split $x=x_{l}+x_{o}+x_{m}+x_{n}$ accordingly. Now we know $\Pi_{l} x=x_{l}$ and 


$$
\begin{aligned}
\Pi_{m} \Pi_{n} x & =\Pi_{m} \Pi_{n}\left(x_{l}+x_{o}+x_{m}+x_{n}\right) \\
& =\Pi_{m} \Pi_{n} x_{l}+\Pi_{m} \Pi_{n} x_{o}+\Pi_{m} \Pi_{n} x_{m}+\Pi_{m} \Pi_{n} x_{n} \\
& =\Pi_{m} \Pi_{n} x_{l}+\Pi_{m} 0+\Pi_{m} x_{m}+\Pi_{m} 0 \\
& =\Pi_{m} \Pi_{n} x_{l} .
\end{aligned}
$$

Because $\left\{P_{\boldsymbol{k}}\right\}_{\boldsymbol{k} \in \mathbb{Z}^{d}}$ is an NSP, we know that $x_{l} \in \operatorname{Ran}\left(P_{l}\right) \subset \operatorname{Ran}\left(P_{n}\right)$ and $x_{l} \in \operatorname{Ran}\left(P_{l}\right) \subset \operatorname{Ran}\left(P_{\boldsymbol{m}}\right)$; hence $\Pi_{m} \Pi_{n} x_{l}=x_{l}$. We conclude that, for arbitrary $x \in X$ holds $\Pi_{m} \Pi_{n} x=x_{l}=\Pi_{l} x$; which proves the lemma.

Theorem 2.14. The two following statements are equivalent:

(1) $\left\{R_{k}\right\}_{k \in \mathbb{Z}^{d}}$ is a CSR, and $\left\{P_{k}\right\}_{k \in \mathbb{Z}^{d}}$ is an NSP; and

(2)

$$
\Pi_{\boldsymbol{m}} \Pi_{\boldsymbol{n}}=\Pi_{\min (\boldsymbol{m}, \boldsymbol{n})} \quad \forall \boldsymbol{m}, \boldsymbol{n} \in \mathbb{Z}^{d}
$$

Proof. The theorem is a direct combination of the three lemmas above.

It is an immediate consequence of the theorem that operators $\Pi_{\boldsymbol{n}}$ associated with commutative transfer operators $\left\{R_{\boldsymbol{k}}\right\}_{\boldsymbol{k} \in \mathbb{Z}^{d}}$ and $\left\{P_{\boldsymbol{k}}\right\}_{\boldsymbol{k} \in \mathbb{Z}^{d}}$ do commute:

$$
\Pi_{m} \Pi_{n}=\Pi_{\min (m, n)}=\Pi_{n} \Pi_{m}
$$

Further, combination of Theorem 2.14 with Theorem 2.9 gives the following corollary, which is a direct generalization of [4, Proposition 2.5]. By the present framework we recognize immediately the essential conditions that lead to this result.

Corollary 2.15. Let $\left\{R_{\boldsymbol{k}}\right\}_{\boldsymbol{k} \in \mathbb{Z}^{d}}$ be a CSR and $\left\{P_{\boldsymbol{k}}\right\}_{\boldsymbol{k} \in \mathbb{Z}^{d}}$ an NSP, then $f_{\boldsymbol{m}}$ and $f_{\boldsymbol{n}}$ are mutually coherent if and only if

$$
\forall \boldsymbol{l} \in \mathbb{Z}^{d} \text { with } \boldsymbol{l} \leqslant \boldsymbol{m}, \boldsymbol{l} \leqslant \boldsymbol{n}, \text { we have } R_{\boldsymbol{l m}} f_{\boldsymbol{m}}=R_{\boldsymbol{l n}} f_{\boldsymbol{n}}
$$

Having seen what the essential structure of commutative transfer operators is, further in this section we assume all transfer operators to be commutative.

\subsection{The merging operator}

Now we have seen how information about a function $u \in X$ can be represented on $V_{\boldsymbol{n}}$, and how the representations $R_{n} u$ are related for different $n \in \mathbb{Z}^{d}$. An important question is how these $R_{n} u$, given for a limited number of $n \in \mathbb{Z}^{d}$, can be used to restore the picture of the original function $u$ as complete as possible.

We start with the situation where information is available from two representations, viz. in $V_{\boldsymbol{n}}, V_{\boldsymbol{m}}$. Therefore we introduce the merging operator $\Pi_{m n}$, which selects for an $x \in X$ the information that can be represented by the combined representations in $V_{n}$ and $V_{m}$.

Definition 2.16. The merging operator $\Pi_{m n}: X \rightarrow X$ is defined by

$$
\Pi_{m n}=\Pi_{m}+\Pi_{n}-\Pi_{\min (\boldsymbol{m}, \boldsymbol{n})}
$$


Lemma 2.17. Let $\left\{R_{n}\right\}$ and $\left\{P_{\boldsymbol{n}}\right\}$ be a set of commutative transfer operators, then:

(1) $\Pi_{m n}=\Pi_{n m}$

(2) if $\boldsymbol{m} \leqslant \boldsymbol{n}$ then $\Pi_{m n}=\Pi_{n}$,

(3) $\Pi_{m n}$ is a projection,

$$
\operatorname{Ran}\left(P_{\boldsymbol{n}}\right) \cap \operatorname{Ran}\left(P_{\boldsymbol{m}}\right)
$$

$\oplus$

(4) $\operatorname{Ran}\left(\Pi_{\boldsymbol{m} \boldsymbol{n}}\right)=\operatorname{Ran}\left(P_{\boldsymbol{n}}\right) \cap \operatorname{Ker}\left(R_{\boldsymbol{m}}\right)$

$\oplus$

$$
\operatorname{Ker}\left(R_{\boldsymbol{n}}\right) \cap \operatorname{Ran}\left(P_{\boldsymbol{m}}\right)
$$

(5) $\operatorname{Ker}\left(\Pi_{m n}\right)=\operatorname{Ker}\left(R_{n}\right) \cap \operatorname{Ker}\left(R_{m}\right)$.

Proof. The first two statements are trivial by the definition of $\Pi_{\boldsymbol{m} \boldsymbol{n}}$. Now set $\boldsymbol{l}=\min (\boldsymbol{m}, \boldsymbol{n})$.

Because $\Pi_{m} \Pi_{n}=\Pi_{n} \Pi_{m}=\Pi_{\min (\boldsymbol{m}, n)}$ we have

$$
\begin{aligned}
\Pi_{m n} \Pi_{m n} & =\left(\Pi_{m}+\Pi_{n}-\Pi_{\min (\boldsymbol{m}, \boldsymbol{n})}\right)\left(\Pi_{m}+\Pi_{n}-\Pi_{\min (m, n)}\right) \\
& =\Pi_{m}+\Pi_{l}-\Pi_{l}+\Pi_{l}+\Pi_{n}-\Pi_{l}-\Pi_{l}-\Pi_{l}+\Pi_{l} \\
& =\Pi_{m}+\Pi_{n}-\Pi_{l}=\Pi_{m n} .
\end{aligned}
$$

Hence, $\Pi_{m n}$ is a projection.

$x \in \operatorname{Ran}\left(\Pi_{\boldsymbol{m} \boldsymbol{n}}\right)$ implies $\exists z$ :

$$
x=\Pi_{\boldsymbol{m} \boldsymbol{n}} z=\Pi_{\boldsymbol{m}} z+\Pi_{\boldsymbol{n}} z-\Pi_{\boldsymbol{l}} z=\left(\Pi_{\boldsymbol{m}}-\Pi_{\boldsymbol{l}}\right) z+\left(\Pi_{\boldsymbol{n}}-\Pi_{\boldsymbol{l}}\right) z+\Pi_{\boldsymbol{l}} z=z_{m}+z_{n}+z_{l} .
$$

It is clear that this is a decomposition according to the direct sum in (18): $z_{l} \in \operatorname{Ran}\left(\Pi_{l}\right) \subset \operatorname{Ran}\left(\Pi_{m}\right) \cap$ $\operatorname{Ran}\left(\Pi_{\boldsymbol{n}}\right) ; z_{m} \in \operatorname{Ran}\left(\Pi_{\boldsymbol{m}}\right)$ and $z_{m} \in \operatorname{Ker}\left(\Pi_{\boldsymbol{n}}\right)$ because

$$
\Pi_{\boldsymbol{n}} z_{m}=\Pi_{\boldsymbol{n}}\left(\Pi_{\boldsymbol{m}}-\Pi_{\boldsymbol{l}}\right)=\Pi_{\min (\boldsymbol{n}, \boldsymbol{m})}-\Pi_{\boldsymbol{l}} z=0
$$

and similarly $z_{n} \in \operatorname{Ran}\left(\Pi_{n}\right) \cap \operatorname{Ker}\left(\Pi_{m}\right)$.

On the other hand, if $z=z_{l}+z_{n}+z_{m}$ is a splitting according to the direct sum, then

$$
\begin{aligned}
& \Pi_{\boldsymbol{l}} z=\Pi_{\boldsymbol{l}}\left(z_{l}+z_{m}+z_{n}\right) \\
& =\Pi_{\boldsymbol{l}} z_{l}+\Pi_{\boldsymbol{n}} \Pi_{\boldsymbol{m}} z_{m}+\Pi_{\boldsymbol{m}} \Pi_{\boldsymbol{n}} z_{n} \\
& =z_{l}+\Pi_{\boldsymbol{n}} z_{m}+\Pi_{\boldsymbol{m}} z_{n}=z_{l}, \\
& \begin{aligned}
\left(\Pi_{\boldsymbol{m}}-\Pi_{\boldsymbol{l}}\right) z & =\left(\Pi_{\boldsymbol{m}}-\Pi_{\boldsymbol{l}}\right)\left(z_{l}+z_{m}+z_{n}\right) \\
& =\left(\Pi_{\boldsymbol{m}}-\Pi_{\boldsymbol{l}}\right) z_{l}+\left(\Pi_{\boldsymbol{m}}-\Pi_{\boldsymbol{l}}\right) z_{m}+\left(\Pi_{\boldsymbol{m}}-\Pi_{\boldsymbol{l}}\right) z_{n} \\
& =z_{l}-z_{l}+\Pi_{\boldsymbol{m}}\left(I-\Pi_{\boldsymbol{n}}\right) z_{m}+\Pi_{\boldsymbol{m}}\left(I-\Pi_{\boldsymbol{n}}\right) z_{n} \\
& =\Pi_{\boldsymbol{m}} z_{m}=z_{m} .
\end{aligned}
\end{aligned}
$$

Analogously $\left(\Pi_{\boldsymbol{n}}-\Pi_{\boldsymbol{l}}\right) z=z_{n}$. Thus

$$
z=z_{l}+z_{m}+z_{n}=\Pi_{l} z+\left(\Pi_{\boldsymbol{m}}-\Pi_{l}\right) z+\left(\Pi_{\boldsymbol{n}}-\Pi_{l}\right) z=\left(\Pi_{\boldsymbol{m}}+\Pi_{\boldsymbol{n}}-\Pi_{l}\right) z=\Pi_{\boldsymbol{m} \boldsymbol{n}} z .
$$


Hence $z \in \operatorname{Ran}\left(\Pi_{m n}\right)$.

Assume that $0=z=z_{l}+z_{n}+z_{m}$ is a splitting as above, then it follows that $0=z_{l}=z_{n}=z_{m}$ because

$$
\begin{aligned}
& 0=\Pi_{l}(z)=\left(\Pi_{m} \Pi_{\boldsymbol{n}}\right)\left(z_{l}+z_{n}+z_{m}\right)=z_{l} \\
& 0=\left(\Pi_{m}-\Pi_{l}\right)(z)=\left(I-\Pi_{\boldsymbol{n}}\right) \Pi_{m}\left(z_{l}+z_{n}+z_{m}\right)=z_{m}
\end{aligned}
$$

and

$$
0=\left(\Pi_{n}-\Pi_{l}\right)(z)=\left(I-\Pi_{m}\right) \Pi_{n}\left(z_{l}+z_{n}+z_{m}\right)=z_{n} .
$$

$\Pi_{m n}=P_{m} R_{m}+P_{n} R_{n}-P_{l} R_{l}$, hence $\operatorname{Ker}\left(\Pi_{m n}\right) \supset \operatorname{Ker}\left(R_{n}\right) \cap \operatorname{Ker}\left(R_{m}\right)$ is trivial.

$\operatorname{Ker}\left(\Pi_{m n}\right) \subset \operatorname{Ker}\left(R_{n}\right) \cap \operatorname{Ker}\left(R_{m}\right)$ is shown as follows. Let $x \in \operatorname{Ker}\left(\Pi_{n m}\right)$, then

$$
0=\Pi_{n m} x=\left(\Pi_{n}-\Pi_{l}\right) x+\left(\Pi_{m}-\Pi_{l}\right) x+\Pi_{l} x=: x_{n}+x_{m}+x_{l} .
$$

This implies $x_{l} \in \operatorname{Ran}\left(\Pi_{l}\right)$ and $x_{n}, x_{m} \in \operatorname{Ker}\left(\Pi_{l}\right)$, so that $x_{l}=0$ and $x_{n}+x_{m}=0$. Further, from

$$
x_{n}=-x_{m} \in \operatorname{Ran}\left(\Pi_{m}\right) \cap \operatorname{Ran}\left(\Pi_{\boldsymbol{n}}\right)=\operatorname{Ran}\left(\Pi_{\min (\boldsymbol{n}, \boldsymbol{m})}\right)=\operatorname{Ran}\left(P_{l}\right)
$$

it follows that $x_{m}=x_{n}=0$, and hence $\operatorname{Ker}\left(\Pi_{m n}\right)=\operatorname{Ker}\left(R_{n}\right) \cap \operatorname{Ker}\left(R_{m}\right)$.

Now we can introduce the hierarchical surplus, $H_{m n} u$, of a function $u$. This hierarchical surplus represents the amount of information in an approximation $u \in V_{\max (\boldsymbol{m}, \boldsymbol{n})}$ that cannot be represented on the $\operatorname{Span}\left(V_{n}, V_{m}\right)$.

Definition 2.18. Let $\left\{R_{n}\right\}$ and $\left\{P_{n}\right\}$ be a commutative set of transfer operators, then we define $H_{\boldsymbol{m} \boldsymbol{n}}: X \rightarrow \operatorname{Ran}\left(P_{\max (\boldsymbol{m}, \boldsymbol{n})}\right) \subset X$, the hierarchical surplus, relative to the grids $\boldsymbol{m}$ and $\boldsymbol{n}$, by

$$
H_{m n}=\Pi_{\max (m, n)}-\Pi_{m n}
$$

Clearly, the hierarchical surplus is a projection operator, and we can write

$$
H_{m n}=\left(\Pi_{\max (n, m)}-\Pi_{n}\right)\left(\Pi_{\max (n, m)}-\Pi_{m}\right) .
$$

This general idea of a hierarchical surplus leads naturally to a partitioning of the spaces $V_{n}$ in more elementary subspaces (pre-wavelet spaces) in the following section.

\subsection{The hierarchical decomposition}

In this section, again, we assume $\left\{R_{n}\right\}$ and $\left\{P_{n}\right\}$ to be commutative sets of transfer operators.

Definition 2.19. For a fixed $n_{0} \in\{-\infty, \mathbb{Z}\}^{d}$, which indicates a coarsest grid, we define for arbitrary $\boldsymbol{n} \geqslant \boldsymbol{n}_{\mathbf{0}}, \boldsymbol{n} \in \mathbb{Z}^{d}$, the operator $Q_{\boldsymbol{n}}: X \rightarrow \operatorname{Ran}\left(P_{\boldsymbol{n}}\right) \subset X$, by

$$
Q_{n}=\prod_{j=1,1, n_{j} \neq n_{0_{j}}}^{d}\left(\Pi_{n}-\Pi_{n-e_{j}}\right) .
$$

We use the convention that $Q_{n_{0}}=\Pi_{n_{0}}$. The operator $Q_{n}$ is called the (direct) hierarchical surplus at grid $\boldsymbol{n}$. 
If $\boldsymbol{n}_{\mathbf{0}} \in \mathbb{Z}^{d}$ we call $\boldsymbol{n}_{\mathbf{0}} \in \mathbb{Z}^{d}$ the coarsest grid, and, without loss of generality we may assume $\boldsymbol{n}_{\mathbf{0}}=\mathbf{0}$. If a $n_{0 j}=-\infty$ then no coarsest grid exists in the $j$ th direction.

Lemma 2.20. $Q_{n}$ is a projection and $Q_{m} Q_{n}=0$ for all $\boldsymbol{m} \neq \boldsymbol{n}$.

Proof. First we show that $Q_{n}$ is a projection. For simplicity of notation, we set $\boldsymbol{c}=n_{\mathbf{0}}$.

$$
\begin{aligned}
Q_{n} Q_{\boldsymbol{n}} & =\prod_{j=1, n_{j} \neq c_{j}}^{d}\left(\Pi_{\boldsymbol{n}}-\Pi_{\boldsymbol{n}-\boldsymbol{e}_{j}}\right)\left(\Pi_{\boldsymbol{n}}-\Pi_{\boldsymbol{n}-\boldsymbol{e}_{j}}\right) \\
& =\prod_{j=1, n_{j} \neq c_{j}}^{d}\left(\Pi_{\boldsymbol{n}}-\Pi_{\boldsymbol{n}-\boldsymbol{e}_{j}}-\Pi_{\boldsymbol{n}-\boldsymbol{e}_{j}}+\Pi_{\boldsymbol{n}-\boldsymbol{e}_{j}}\right)=\prod_{j=1, n_{j} \neq c_{j}}^{d}\left(\Pi_{\boldsymbol{n}}-\Pi_{\boldsymbol{n}-\boldsymbol{e}_{j}}\right)=Q_{\boldsymbol{n}} .
\end{aligned}
$$

To show that $Q_{m} Q_{n}=0$ for all $\boldsymbol{m} \neq \boldsymbol{n}$, let $\boldsymbol{m} \neq \boldsymbol{n}$. Without loss of generality we may assume $n_{i}<m_{i}$ for some $i \in\{1, \ldots, d\}$. We consider the case $n_{i} \neq c_{i}$; the other cases are similar.

$$
\begin{aligned}
Q_{\boldsymbol{m}} Q_{\boldsymbol{n}} & =\prod_{j}\left(\Pi_{\boldsymbol{m}}-\Pi_{\boldsymbol{m}-\boldsymbol{e}_{j}}\right)\left(\Pi_{\boldsymbol{n}}-\Pi_{\boldsymbol{n}-\boldsymbol{e}_{j}}\right) \\
& =\left(\Pi_{\boldsymbol{m}}-\Pi_{\boldsymbol{m}-\boldsymbol{e}_{i}}\right)\left(\Pi_{\boldsymbol{n}}-\Pi_{\boldsymbol{n}-\boldsymbol{e}_{i}}\right) \prod_{j \neq i} \ldots \\
& =\left(\Pi_{\left(\ldots, n_{l}, \ldots\right)}-\Pi_{\left(\ldots, n_{l}, \ldots\right)}-\Pi_{\left(\ldots, n_{l}-1, \ldots\right)}+\Pi_{\left(\ldots, n_{l}-1, \ldots\right)}\right) \prod_{j \neq i} \ldots=0 .
\end{aligned}
$$

The indices indicated by dots correspond with those of $\min (\boldsymbol{n}, \boldsymbol{m})$.

Notice that, for $\boldsymbol{n}>\boldsymbol{n}_{\mathbf{0}}$, the two-dimensional case the relation (21) reads

$$
Q_{n} u=\Pi_{n} u-\Pi_{n-e_{1}} u-\Pi_{n-e_{2}} u+\Pi_{n-e} u,
$$

where $e=(1,1)$, and in the one-dimensional case we have

$$
Q_{n} u=\Pi_{n} u-\Pi_{n-\boldsymbol{e}} u .
$$

Corollary 2.21. From Definition 2.19 it is immediately obvious that the projection $\Pi_{n}$ can be decomposed as

$$
\Pi_{n}=\sum_{n_{0} \leqslant m \leqslant n} Q_{m}
$$

and, hence, $\operatorname{Ran}\left(\Pi_{n}\right)=\operatorname{Span}\left(\operatorname{Ran}\left(Q_{m}\right)\right)_{n_{0} \leqslant m \leqslant n}$. Because of Lemma 2.20 we can write

$$
\operatorname{Ran}\left(\Pi_{\boldsymbol{n}}\right)=\bigoplus_{\boldsymbol{n}_{\mathbf{0}} \leqslant \boldsymbol{m} \leqslant \boldsymbol{n}} \operatorname{Ran}\left(Q_{\boldsymbol{m}}\right)
$$

If $V_{\boldsymbol{n}} \subset X$ and $P_{\boldsymbol{n}}$ is the natural injection, then we see that $\operatorname{Ran}\left(P_{n}\right)=\operatorname{Ran}\left(\Pi_{\boldsymbol{n}}\right)=V_{\boldsymbol{n}}$ and, defining the pre-wavelet space $W_{n}=\operatorname{Ran}\left(Q_{m}\right)$, we find

$$
V_{n}=\bigoplus_{n_{0} \leqslant m \leqslant n} W_{m}
$$


Notice that, given the set $\left\{W_{m}\right\}_{n_{0} \leqslant m}$, the expansion (28) essentially describes the complete partially ordered set of approximating spaces. In fact, if we can write $X=\bigoplus_{n_{0} \leqslant m} W_{m}$, we induce automatically a structure for prolongations and restrictions, which-now by nature-form a commutative and hence nested sequence.

\subsection{The tensor product case}

In this section we introduce the usual and by far the most interesting examples of commutative sets of operators, constructed by means of tensor products. Of course it is also possible to constructby trivial means-cases where (28) is no tensor product space, e.g., by taking arbitrary linearly independent spaces $W_{m}$. Although such non-tensor product spaces maybe useful in particular cases, tensor product spaces give more regular and interesting examples.

For any $i=1, \ldots, d$, let $\Omega_{i} \subset \mathbb{R}$ and let $\Omega=\bigotimes_{i=1}^{d} \Omega_{i} \subset \mathbb{R}^{d}$ be their Cartesian product. Let $X_{i}\left(\Omega_{i}\right)$ be a function space on $\Omega_{i}$ with functions $u_{i, a_{i}}\left(x_{i}\right)$ so that $X_{i}\left(\Omega_{i}\right)=\operatorname{Span}\left(\left\{u_{i, a_{i}}\right\}_{a_{i} \in A_{i}}\right)$, for some index set $A_{i}$. Let $A=\bigotimes_{i=1}^{d} A_{i}$ be the Cartesian product of index sets. Then the tensor product space $X(\Omega)$ is defined by

$$
X(\Omega)=\bigotimes_{i=1}^{d} X_{i}\left(\Omega_{i}\right)=\underset{\left(a_{1}, a_{2}, \ldots, a_{d}\right) \in A}{\operatorname{Span}} \prod_{i=1}^{d} u_{i, a_{i}} .
$$

It is well known, e.g., that for $X_{i}\left(\Omega_{i}\right)=C_{0}^{\infty}(\mathbb{R})$, the tensor product space $X(\Omega)$ is densely embedded both in $C^{l}\left(\mathbb{R}^{d}\right)$ and in $H^{l}\left(\mathbb{R}^{d}\right)$.

For each $i=1, \ldots, d$, let $\left\{R_{i, n}\right\}_{n \in \mathbb{Z}}$ be a sequence of restrictions (for functions in one dimension) defined on $X_{i}\left(\Omega_{i}\right)$, with $R_{i, n}: X_{i}\left(\Omega_{i}\right) \rightarrow V_{i, n}\left(\Omega_{i}\right) \subset X_{i}\left(\Omega_{i}\right)$, then, similar to $X(\Omega)$, for each $\boldsymbol{n} \in \mathbb{Z}^{d}$ we may define a tensor product space $V_{n}(\Omega)=\bigotimes_{i=1}^{d} V_{i, n_{i}}\left(\Omega_{i}\right)$.

If the elements of $V_{i, n_{i}}\left(\Omega_{i}\right)$ are all determined by values associated with $\Omega_{n_{i}}\left(\Omega_{n_{i}}^{+}\right.$or $\left.\Omega_{n_{i}}^{*}\right)$, then we find a bijection $V_{n}=V_{n}(\Omega) \cong V_{n}\left(\Omega_{n}\right)$ (or $\cong V_{n}\left(\Omega_{n}^{+}\right)$or $\cong V_{n}\left(\Omega_{n}^{*}\right)$ ). This notation indicates that its elements are determined by their values on the Cartesian product space $\Omega_{n}=\bigotimes_{i=1}^{d} \Omega_{n_{i}}$ (or $\Omega_{n}^{+}$or $\Omega_{n}^{*}$ ).

Definition 2.22. We define the tensor product restriction $R_{n}: X(\Omega) \rightarrow V_{n}(\Omega)$ by its action on a typical basis function

$$
u_{\boldsymbol{a}}(\boldsymbol{x})=\prod_{i=1}^{d} u_{i, a_{i}}\left(x_{i}\right) \mapsto R_{\boldsymbol{n}} u_{\boldsymbol{a}}(\boldsymbol{x})=\prod_{i=1}^{d} R_{i, n_{i}} u_{i, a_{i}}\left(x_{i}\right) .
$$

We also write $R_{n}=\bigotimes_{i} R_{i, n_{i}}$. Since $V_{n}(\Omega) \subset X(\Omega)$, we can take the natural injection $P_{n}$ as the corresponding reconstruction for $R_{n}$. This $P_{n}$ we call the tensor product prolongation.

Theorem 2.23. For each $i \in\{1, \ldots, d\}$ let $\Omega_{i} \subset \mathbb{R}$, and let $\left\{V_{i, n}\left(\Omega_{i}\right)\right\}_{n \in \mathbb{Z}}$ form a nested sequence of subspaces of the function space $X\left(\Omega_{i}\right)$, with

$$
V_{i, p}\left(\Omega_{i}\right) \subset V_{i, q}\left(\Omega_{i}\right) \subset X\left(\Omega_{i}\right) \text { for } p \leqslant q,
$$

and let each sequence of (one-dimensional) operators $\left\{R_{i, n}\right\}_{n \in \mathbb{Z}}$ form an NSR. If we take for the corresponding reconstructions $\left\{P_{i, n}\right\}_{n \in \mathbb{Z}}$ the natural injection, then the tensor product restrictions $\left\{R_{n}\right\}$ 
and prolongations $\left\{P_{n}\right\}$ form a CSR and a CSP, respectively. (So, together they form a commutative set of transfer operators.)

Proof. By the nesting of the subspaces it is immediate that for each $i$ the set $\left\{P_{i, n}\right\}_{n \in \mathbb{Z}}$ forms an NSP (Section 2.1). Further, using the fact that the prolongation is the natural injection, we can identify restrictions $R_{i}$ with the corresponding projections $\Pi_{i}$, and by Lemma 2.7 we see that for each $i$ we have $\Pi_{i, p} \Pi_{i, q}=\Pi_{i, q} \Pi_{i, p}=\Pi_{i, p}$ if $p \leqslant q$. Thus, for each $i$ it follows that the sequences $\left\{P_{i, n}\right\}_{n \in \mathbb{Z}}$ and $\left\{R_{i, n}\right\}_{n \in \mathbb{Z}}$ are CSP and SCR. Now we prove that $\left\{R_{\boldsymbol{n}}\right\}$ is an CSR by showing $R_{m} R_{n}=R_{\min (\boldsymbol{m}, \boldsymbol{n})}$ as follows.

Let $\boldsymbol{l}=\min (\boldsymbol{m}, \boldsymbol{n})$ and let $v \in X(\Omega)$ be arbitrary, then we can write

$$
\begin{aligned}
& v(\boldsymbol{x})=\sum_{\boldsymbol{a} \in A} c_{\boldsymbol{a}} u_{\boldsymbol{a}}(\boldsymbol{x}), \\
& R_{\boldsymbol{m}} v(\boldsymbol{x})=\sum_{\boldsymbol{a}} c_{\boldsymbol{a}} R_{\boldsymbol{m}} u_{\boldsymbol{a}}(\boldsymbol{x})=\sum_{\boldsymbol{a}} c_{\boldsymbol{a}} \prod_{i} R_{i, m_{i}} u_{i, a_{i}}\left(x_{i}\right) .
\end{aligned}
$$

Similarly,

$$
\begin{aligned}
R_{\boldsymbol{n}} R_{\boldsymbol{m}} v(\boldsymbol{x}) & =\sum_{\boldsymbol{a}} c_{\boldsymbol{a}} R_{\boldsymbol{n}} \prod_{i} R_{i, m_{i}} u_{i, a_{i}}\left(x_{i}\right)=\sum_{\boldsymbol{a}} c_{\boldsymbol{a}} \prod_{i} R_{i, n_{i}} R_{i, m_{i}} u_{i, a_{i}}\left(x_{i}\right) \\
& =\sum_{\boldsymbol{a}} c_{\boldsymbol{a}} \prod_{i} R_{i, \min \left(m_{i}, n_{i}\right)} u_{i, a_{i}}\left(x_{i}\right)=\sum_{\boldsymbol{a}} c_{\boldsymbol{a}} R_{\min (\boldsymbol{m}, \boldsymbol{n})} \prod_{i} u_{i, a_{i}}\left(x_{i}\right) \\
& =R_{\min (\boldsymbol{m}, \boldsymbol{n})} \sum c_{\boldsymbol{a}} \prod_{i} u_{i, a_{i}}\left(x_{i}\right)=R_{\min (\boldsymbol{m}, \boldsymbol{n})} v(\boldsymbol{x}) .
\end{aligned}
$$

Hence $R_{\boldsymbol{n}} R_{\boldsymbol{m}}=R_{\min (\boldsymbol{m}, \boldsymbol{n})}$, and, thus, $\Pi_{\boldsymbol{n}} \Pi_{\boldsymbol{m}}=\Pi_{\min (\boldsymbol{m}, \boldsymbol{n})}$. Now Theorem 2.14 shows that $\left\{R_{\boldsymbol{n}}\right\}$ is an CSR and $\left\{P_{\boldsymbol{n}}\right\}$ is an NSP.

To prove that $\left\{P_{\boldsymbol{n}}\right\}$ is an CSP, we have to show $V_{\min (\boldsymbol{m}, \boldsymbol{n})}=V_{\boldsymbol{m}} \cap V_{\boldsymbol{n}}$, or

$$
\bigotimes_{i} V_{i, \min \left(m_{i}, n_{i}\right)}=\bigotimes_{i} V_{i, m_{i}} \cap \bigotimes_{i} V_{i, n_{i}}
$$

As for each $i$ we know that $\left\{V_{i, n}\left(\Omega_{i}\right)\right\}_{n \in \mathbb{Z}}$ is a nested sequence of subspaces of $X_{i}\left(\Omega_{i}\right)$, we can construct a sequentially ordered set of basis functions $B_{i}=\left\{u_{i, b}\right\}_{b}$ in $X_{i}\left(\Omega_{i}\right)$, such that $n_{i}<m_{i}$ implies $u_{i, b} \in V_{i, n_{i}} \Rightarrow u_{i, b} \in V_{i, m_{i}}$. It follows that we have $V_{i, m_{i}}=\operatorname{Span}\left(\left\{u_{i, l} \in B_{i} \mid u_{i, l} \in V_{i, m_{i}}\right\}\right)$ and similarly

$$
V_{i, \min \left(n_{i}, m_{i}\right)}=\operatorname{Span}\left(\left\{u_{i, l} \in B_{i} \mid u_{i, l} \in V_{i, n_{i}} \cap V_{i, m_{i}}\right\}\right) .
$$

So we see

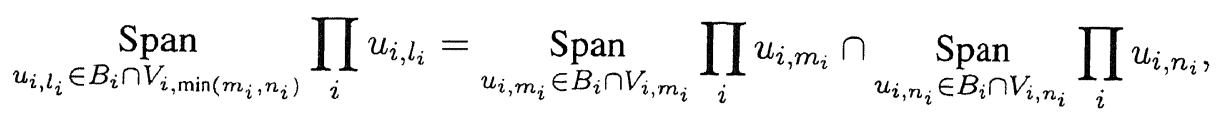

which is equivalent with (32).

Example 2.24 (Piecewise constant approximation). If we consider $L_{2}^{\text {loc }}(\Omega)=\overline{X(\Omega)}$ and we choose for $R_{i, n}$ the one-dimensional $L_{2}$-projection $R_{i, n}: X\left(\Omega_{i}\right) \rightarrow V_{n}\left(\Omega_{i}\right) \subset X\left(\Omega_{i}\right)$, where $\Omega_{i} \subset \mathbb{R}$ and 
$V_{n}\left(\Omega_{i}\right)$ is the space of piecewise constant functions on dyadic intervals (i.e., if $R_{i, n}$ denotes taking mean values over intervals $\left[j 2^{-n},(j+1) 2^{-n}\right]$ in the $i$ th coordinate direction), then, for each $i$, the restrictions $\left\{R_{i, n}\right\}_{n}$ form a one-dimensional NSR. The corresponding reconstructions $P_{i, n}$ represent piecewise constant interpolation over dyadic intervals. This makes the prolongations $\left\{P_{i, n}\right\}_{n}$ an NSP.

Then, as a consequence of the above theorem, $\left\{R_{k}\right\}$ and $\left\{P_{k}\right\}$ are commutative transfer operators, i.e., $\left\{R_{\boldsymbol{k}}\right\}$ is a CSR and $\left\{P_{\boldsymbol{k}}\right\}$ a CSP.

Example 2.25 (Piecewise $d$-linear approximation). If we select the restriction $R_{i, n}: C^{0}\left(\Omega_{i}\right) \rightarrow$ $V_{n}\left(\Omega_{i}\right) \subset \mathbb{R}^{\mathbb{Z}}$ to be taking function values at dyadic points $j 2^{-n}$ in the interval $\Omega_{i} \subset \mathbb{R}$, then $\left\{R_{i, n}\right\}_{n}$ is an NSR. Corresponding reconstructions $P_{i, n}$, defined by piecewise linear interpolation over dyadic intervals, make the prolongations $\left\{P_{i, n}\right\}_{n}$ an NSP.

As a consequence of the previous theorem, with $C^{0}(\Omega)=\overline{X(\Omega)}$, the tensor product operators $\left\{R_{\boldsymbol{k}}\right\}$ and $\left\{P_{k}\right\}$, defined on $X(\Omega)$, are commutative (are a CSR and a CSP respectively). The restriction $R_{k}$ takes the function values at grid points $\Omega_{n}^{+}$, and the prolongation $P_{k}$ makes a multi-linear interpolation over cells in $\Omega_{n}$.

In the above examples, with $V_{n} \subset X$ we took for the reconstruction $P_{n}$ the natural injection (the identity in $X$ ). In this way we may identify $R_{n}$ and $\Pi_{n}$. It appears that in both cases, i.e., for the piecewise constant and the piecewise linear approximation, we have a projection $\Pi_{n}$ of the form

$$
\Pi_{n}=\prod_{j=1}^{d} \Pi_{n_{j} \boldsymbol{e}_{j}}=\prod_{j=1}^{d} R_{n_{j} e_{j}} .
$$

Here $R_{n_{j} e_{j}}: X(\Omega) \rightarrow X(\Omega)$ is the operator on the tensor product space $X(\Omega)$ such that

$$
R_{n_{j} \boldsymbol{e}_{j}} u_{\boldsymbol{a}}(\boldsymbol{x})=R_{j, n_{j}} u_{j, a_{j}}\left(x_{j}\right) \cdot \prod_{i \neq j}^{d} u_{i, a_{i}}\left(x_{i}\right) .
$$

In the following section we consider the case of nested subspaces $\left\{V_{n}\right\}$ with $V_{n} \subset X$ and $X=$ $\overline{\bigcup_{n} V_{n}}$, and where all spaces $V_{n}$ are spanned by dilations of a single function $\phi(\boldsymbol{x})$, together with all its dyadic translations. This leads to the more-dimensional multiresolution analysis or MRA. In this case the spaces $W_{m}=\operatorname{Ran}\left(Q_{m}\right)$ correspond with more-dimensional wavelet spaces.

\subsection{More-dimensional MRA and wavelets}

It will be convenient if

(i) we can make an arbitrarily accurate approximation of any function $u \in X$ by taking the multi-integer $n$ large enough.

Moreover, it will be convenient if

(ii) all spaces $\left\{\operatorname{Ran}\left(P_{n}\right)\right\}$ or $\left\{V_{n}\right\}$ have a similar structure, and

(iii) there is a clear relation between the spaces in $\left\{\operatorname{Ran}\left(P_{n}\right)\right\}$ or $\left\{V_{n}\right\}$. 
In order to create such a structure, in this section we introduce the multidimensional multiresolution analysis. For this purpose we will restrict ourselves to Hilbert spaces. First we introduce the important notion of frame.

Definition 2.26. A sequence $\left\{x_{n}\right\}$ in a Hilbert space $H$ is a frame if there exist numbers $A, B>0$ such that for all $x \in H$ we have

$$
A\|x\|^{2} \leqslant \sum_{n}\left|\left(x, x_{n}\right)\right|^{2} \leqslant B\|x\|^{2} .
$$

The numbers $A, B$ are called frame bounds. The frame is tight if $A=B$. The frame is exact if it ceases to be a frame whenever any single element is deleted from the sequence. If the sequence $\left\{x_{n}\right\}$ satisfies (only) the second part of the inequality (33) then the sequence is called a Bessel sequence.

Having introduced the exact frame, we can define the partially ordered, more-dimensional multiresolution analysis. Notice that this is different from the more-dimensional multiresolution analysis introduced in [1], which considers a sequentially ordered nested set of approximating spaces.

Definition 2.27. Let $\Omega=\mathbb{R}^{d}$ and let $X(\Omega)$ be a Hilbert space of functions defined on $\Omega$. A multidimensional multiresolution analysis of $X(\Omega)$, is a partially ordered set of closed linear subspaces

$$
\left\{V_{n} \mid V_{n} \subset X(\Omega)\right\}_{n \in \mathbb{Z}^{d}}
$$

with the four properties:

$$
\begin{aligned}
& \bigcap_{\boldsymbol{n}} V_{\boldsymbol{n}}=\{0\} ; \quad \overline{\bigcup_{\boldsymbol{n}} V_{\boldsymbol{n}}}=X(\Omega), \\
& f(\boldsymbol{x}) \in V_{\boldsymbol{n}} \Leftrightarrow f\left(2^{\boldsymbol{m}} \boldsymbol{x}\right) \in V_{\boldsymbol{n}+\boldsymbol{m}} \quad \forall \boldsymbol{n}, \boldsymbol{m} \in \mathbb{Z}^{d}, \\
& f(\boldsymbol{x}) \in V_{\boldsymbol{n}} \Leftrightarrow f\left(\boldsymbol{x}-2^{-\boldsymbol{n}} \boldsymbol{k}\right) \in V_{\boldsymbol{n}} \quad \forall \boldsymbol{n}, \boldsymbol{k} \in \mathbb{Z}^{d}, \\
& \exists \phi \in V_{\mathbf{0}}: \quad\{\phi(\boldsymbol{x}-\boldsymbol{k})\}_{\boldsymbol{k} \in \mathbb{Z}^{d}} \text { is an exact frame for } V_{\mathbf{0}} .
\end{aligned}
$$

The function $\phi(\boldsymbol{x})$ in $(34 \mathrm{~d})$ is called the father function or the scaling function of the multiresolution analysis.

For $\Omega=\mathbb{R}^{d}$ the tensor product Examples 2.24 and 2.25 in Section 2.5 also yield examples of a multidimensional MRA.

For piecewise constant interpolation we take $X(\Omega)=L^{2}\left(\mathbb{R}^{d}\right)$ as the starting point. The characteristic function on the unit cube (the more-dimensional Haar function) is the scaling function $\phi$. The set $\left\{V_{n}\right\}$ contains the spaces of piecewise constant functions on $\Omega_{n}$, and a CSR is obtained by $R_{n}: X(\Omega) \rightarrow V_{n}$, the $L^{2}$-projection. It is obvious that in this case the set $\{\phi(\boldsymbol{x}-\boldsymbol{k})\}_{\boldsymbol{k}}$ is an orthonormal basis and hence an exact frame with bounds $A=B=1$.

For piecewise linear interpolation we take $X(\Omega)=H^{e}\left(\mathbb{R}^{d}\right)$ as the Hilbert space. The set $\left\{V_{n}\right\}$ contains the space of piecewise $d$-linear functions, determined by their nodal values at $\Omega_{n}^{+}$. A CSR is obtained by $R_{n}: X(\Omega) \rightarrow V_{n}$, the piecewise $d$-linear interpolation at $\Omega_{n}^{+}$. Here, the $d$-linear finiteelement basis function is the scaling function $\phi$. By [9, Theorem 2.1.3] it is easily seen that in this case $\{\phi(\boldsymbol{x}-\boldsymbol{k})\}_{\boldsymbol{k}}$ is an exact frame, with as frame bounds the extreme eigenvalues of the frame 
operator $S: L^{2}(\Omega) \rightarrow L^{2}(\Omega)$ defined by $S u=\sum_{\boldsymbol{n}}(u, \phi(\boldsymbol{x}-\boldsymbol{k})) \phi(\boldsymbol{x}-\boldsymbol{k})$. Bounds for these extreme eigenvalues are $A=3^{-d}$ and $B=1$, respectively.

As in the tensor product case, we take for the reconstruction the natural injection $P_{n}: V_{n} \rightarrow X$ so that $R_{n} \equiv \Pi_{n}$ for all $n \in \mathbb{Z}^{d}$.

\section{More-dimensional wavelets}

A wavelet space $W_{n} \subset V_{n}$, a closed subspace of $V_{n}$ which contains those functions in $V_{n}$ that cannot be represented in any of the function spaces on the next coarser level, i.e., these functions are in $V_{n}$ but not in $\operatorname{Span}\left(V_{n-e_{1}}, \ldots, V_{n-e_{d}}\right)$. Thus $W_{n} \subset V_{n}$ is a closed subspace so that

$$
V_{n}=W_{n} \oplus \operatorname{Span}\left(V_{n-e_{1}}, \ldots, V_{n-e_{d}}\right) \text {. }
$$

This means that $W_{n}$ contains the 'difference information' that is available in the fine grid $V_{n}$ but not in the span of the coarser grids $V_{n-e_{1}}, V_{n-e_{2}}, \ldots, V_{n-e_{d}}$.

The space $W_{\boldsymbol{n}}$ is the complement of $\operatorname{Span}\left(V_{n-e_{1}}, \ldots, V_{\boldsymbol{n}-e_{d}}\right)$ in $V_{\boldsymbol{n}}$. Of course, this complement is not uniquely determined. If we want we can make use of the Hilbert space structure and consider the (unique) orthogonal complement

$$
W_{\boldsymbol{n}} \perp \operatorname{Span}\left(V_{\boldsymbol{n}-\boldsymbol{e}_{1}}, \ldots, V_{\boldsymbol{n}-\boldsymbol{e}_{d}}\right) \text {. }
$$

This choice corresponds with $R_{n}: X \rightarrow V_{n}$ being the orthogonal projection. However, in many cases we will use spaces $W_{n}$ that don't satisfy this orthogonality property!

As soon as we have selected a CSR $\left\{R_{n}\right\}$, then corresponding pre-wavelet spaces are defined as in Section 2.4. These pre-wavelet spaces on an MRA are wavelet spaces.

In the case of an MRA no coarsest grid exists, so that (28) gives

$$
V_{n}=\bigoplus_{j \leqslant n} W_{j}
$$

Because of property (34a) we can decompose the space $X(\Omega)$ in

$$
X(\Omega)=\bigoplus_{\boldsymbol{j} \in \mathbb{Z}^{d}} W_{j}
$$

so that we can write any $u \in X(\Omega)$ as $u=\sum_{\boldsymbol{j} \in \mathbb{Z}^{d}} w_{\boldsymbol{j}}$ with $w_{\boldsymbol{j}} \in W_{\boldsymbol{j}}$. A restriction $R_{\boldsymbol{n}}: X(\Omega) \rightarrow V_{\boldsymbol{n}}$ is now determined by

$$
R_{n}\left(\sum_{j} w_{j}\right)=\sum_{j \leqslant n} w_{j}
$$

By Definition 2.19 we recognize the direct hierarchical surplus

$$
Q_{n}: X(\Omega) \rightarrow \operatorname{Ran}\left(Q_{n}\right)=W_{n} .
$$

We see that there is no coarsest grid and we can decompose $R_{n}$ as

$$
R_{n}=\sum_{k \leqslant n} Q_{k}
$$

The four relations (34a)-(34d) imply that also the spaces $W_{\boldsymbol{n}}$ are scaled versions of one space $W_{\mathbf{0}}$,

$$
f(\boldsymbol{x}) \in W_{\boldsymbol{n}} \Leftrightarrow f\left(2^{-n} \boldsymbol{x}\right) \in W_{\mathbf{0}}, \quad \forall \boldsymbol{n} \in \mathbb{Z}^{d},
$$


and, moreover, that they are translation invariant for the discrete translations $2^{-n} \mathbb{Z}^{d}$,

$$
f(\boldsymbol{x}) \in W_{\mathbf{0}} \Leftrightarrow f(\boldsymbol{x}-\boldsymbol{k}) \in W_{\mathbf{0}}, \quad \forall \boldsymbol{n} \in \mathbb{Z}^{d} .
$$

As soon as we find a function $\psi(\boldsymbol{x})$ with the property that $\psi(\boldsymbol{x}-\boldsymbol{k}), k \in \mathbb{Z}^{d}$, is a basis of $W_{\boldsymbol{e}}$, then by a simple rescaling we see that $\psi\left(2^{n} \boldsymbol{x}-\boldsymbol{k}\right)$, yields a basis of $W_{n+e}$. Such a function is the more-dimensional generalization of a wavelet [3]. Because of (38) the full collection

$$
\left\{\psi_{\boldsymbol{n}, \boldsymbol{k}}(\boldsymbol{x}) \mid \psi_{\boldsymbol{n}, \boldsymbol{k}}(\boldsymbol{x})=\psi\left(2^{n} \boldsymbol{x}-\boldsymbol{k}\right), \boldsymbol{n}, \boldsymbol{k} \in \mathbb{Z}^{d}\right\}
$$

is a basis of $X\left(\mathbb{R}^{d}\right)$.

\section{Piecewise approximation in $d$ dimensions}

In this section, we first describe the approximating function spaces for piecewise constant and piecewise multilinear approximation. For the spaces $V_{n}$ and for the pre-wavelet spaces $W_{n}$ we describe basis functions. Using these bases, in Sections 3.2 and 3.3 we give proofs for error estimates on regular and sparse grids. Most of the estimates are essentially also found in $[2,13,16]$, but here the proofs are more general, simpler and given in a unified treatment.

\subsection{Piecewise approximation}

\subsubsection{Piecewise constant approximation}

First, let $\Omega=\mathbb{R}^{d}$ or $\Omega=(0,1)^{d}$. We approximate $u \in X(\Omega)=L_{\text {loc }}^{p}(\Omega)$ by $u_{n} \in V_{n}$, in the space of piecewise constant functions on $\Omega_{\boldsymbol{n}}$, i.e., in

$$
V_{\boldsymbol{n}}=\operatorname{Span}\left(\left\{\phi_{\boldsymbol{n} j}\right\}\right)
$$

with, for some $q \geqslant 1$ or $q=\infty$,

$$
\begin{aligned}
& \phi_{\boldsymbol{n} \boldsymbol{j}}(\boldsymbol{x})=2^{|\boldsymbol{n}| / q} \phi\left(2^{\boldsymbol{n}} \boldsymbol{x}-\boldsymbol{j}\right) \\
& \phi(\boldsymbol{x})=\prod_{j=1}^{d} \chi_{[0,1]}\left(x_{j}\right), \quad \text { with } \chi_{[0,1]}(x) \text { the characteristic function on the unit interval. }
\end{aligned}
$$

This clearly describes a basis for a tensor product space, and we may write

$$
V_{\boldsymbol{n}}=V_{\boldsymbol{n}}(\Omega)=\bigotimes_{j=1}^{d} V_{n_{j}}\left(\Omega_{j}\right),
$$

the tensor product of spaces $V_{n_{j}}\left(\Omega_{j}\right)$. These $V_{n_{j}}$ are the spaces of piecewise constant functions with meshwidth $h_{j}=2^{-n_{j}}$ on $\Omega_{j} \subset \mathbb{R}$. The corresponding grid of cells on the Cartesian product of $\left\{\Omega_{j}\right\}$, is denoted by $\Omega_{n}$. The cell centers are denoted by by $\Omega_{n}^{*}$.

We define the restriction $R_{n}$ as the projection

$$
\begin{aligned}
R_{\boldsymbol{n}}: X & \rightarrow V_{\boldsymbol{n}} \subset X, \\
u & \mapsto u_{\boldsymbol{n}}=R_{\boldsymbol{n}} u, \quad \text { with } u_{\boldsymbol{n}, \boldsymbol{i}}=u_{\boldsymbol{n}}((\boldsymbol{i}+\boldsymbol{e} / 2) \boldsymbol{h})=2^{+|\boldsymbol{n}|} \int_{\Omega_{\boldsymbol{n} i}} u(\xi) \mathrm{d} \Omega .
\end{aligned}
$$


This restriction is of type (30), and $R_{n}=R_{n_{1}, \ldots, n_{d}}$ can be decomposed as

$$
R_{n}=\prod_{j=1}^{d} R_{n_{j} e_{j}}
$$

where $R_{n_{j}} e_{j} u(x)$ is the function, piecewise constant in the $j$ th coordinate direction on a partitioning $\Omega_{n}$, so that

$$
R_{n_{j} \boldsymbol{e}_{j}} u(\boldsymbol{x})=2^{n_{j}} \int_{\boldsymbol{x}-(h / 2) \boldsymbol{e}_{j}}^{\boldsymbol{x}+(h / 2) \boldsymbol{e}_{j}} u\left(\xi_{1}, \ldots, \xi_{d}\right) \mathrm{d} \xi_{j}
$$

for all $\boldsymbol{x}$ with $\left(x_{j} 2^{n_{j}} \pm 1 / 2\right) \in \mathbb{Z}$.

In the special case $X=L^{2}(\Omega)$, the space $X=X(\Omega)$ is a Hilbert space, and $\left\{\phi_{n j}\right\}$ is an orthogonal (orthonormal if $q=2$ ) basis in $V_{n}$. In this case $R_{n}$ is the orthogonal projection $L^{2}(\Omega) \rightarrow V_{n}$. For $\Omega=\mathbb{R}^{d}$, the set $\left\{V_{n}\right\}$ as defined in (44)-(45) is a typical MRA. This is no longer the case if we consider a bounded domain $\Omega$, but the decomposition as treated in Section 2 still can be used in the case of a bounded domain.

It is easily checked that the more-dimensional wavelet $\psi(\boldsymbol{x}) \in W_{\boldsymbol{e}}$, corresponding with the piecewise constant scaling function $\phi(x) \in V_{\mathbf{0}}$, from the previous section, is the more-dimensional elementary checkerboard function given by

$$
\psi(\boldsymbol{x})= \begin{cases}0, & \text { if } \boldsymbol{x} \notin \Omega_{\mathbf{0}, \mathbf{0}}, \\ (-1)^{|\boldsymbol{k}|}, & \text { if } \boldsymbol{x} \in \Omega_{\mathbf{0}, \mathbf{0}} \text { and } \boldsymbol{x} \in \Omega_{\boldsymbol{e}, \boldsymbol{k}} .\end{cases}
$$

This function is the tensor product of the Haar wavelet.

In wavelet theory the spaces $W_{n}$ are labeled channels, and the distinct channels are linearly independent. The first decomposition of an arbitrary function from $X(\Omega)$ consists in writing $u(\boldsymbol{x})=$ $\sum_{n} w_{\boldsymbol{n}}(\boldsymbol{x})$, where $w_{\boldsymbol{n}} \in W_{n}$ with $\boldsymbol{n} \in \mathbb{Z}^{d}$, according to (38).

Each subspace $W_{n}$, has its natural basis, the standard basis, ${ }^{3}$

$$
\left\{\psi_{n, k}(\boldsymbol{x}) \mid \psi_{n, k}(\boldsymbol{x})=2^{|\boldsymbol{n}-\boldsymbol{e}| / q} \psi\left(2^{n-e} \boldsymbol{x}-\boldsymbol{k}\right), \boldsymbol{k} \in \mathbb{Z}^{d}\right\},
$$

of functions with a minimal support. We see that $\psi=\psi_{\boldsymbol{e}, 0} \in V_{\boldsymbol{e}}$ is a function with the unit cube $\Omega_{\mathbf{0}, \mathbf{0}}$ as support. The basis function $\psi_{\boldsymbol{n}, \boldsymbol{k}}$ is a scaled, elementary checkerboard function, that may be characterized either by its support, which is a single cell in $\Omega_{n-e}$, or by the centerpoint of this cell, $z_{n-e, k}=2^{-|n-e|}(\boldsymbol{k}+e / 2)$.

On the open unit cube $\Omega=(0,1)^{d}$ we consider the $2^{|n|}$-dimensional spaces $V_{n}=\bigotimes_{j=1}^{d} V_{n_{j}}$, the tensor product of $V_{n_{j}}((0,1))$, the spaces of piecewise constant functions with meshwidth $h_{j}=2^{-n_{j}}$ in the $j$ th coordinate direction. For functions defined on $\Omega=(0,1)^{d}$ we can write relation (38) as

$$
X(\Omega)=\bigoplus_{n \geqslant 0} W_{n}
$$

\footnotetext{
${ }^{3}$ Notice that in more dimensions we use the indexing $\psi_{n+\boldsymbol{e}, \boldsymbol{k}}$, whereas in the one-dimensional case one usually writes $\psi_{k}^{n}$.
} 
and make a decomposition in channels correspondingly. Each subspace $W_{\boldsymbol{n}+\boldsymbol{e}}$, now with $\boldsymbol{n} \geqslant \mathbf{0}$, has its standard basis $\psi_{n+e, k}$ :

$$
\left\{\psi_{\boldsymbol{n}+\boldsymbol{e}, \boldsymbol{k}}(\boldsymbol{x}) \mid \psi_{\boldsymbol{n}+\boldsymbol{e}, \boldsymbol{k}}(\boldsymbol{x})=2^{|\boldsymbol{n}| / q} \psi\left(2^{n} \boldsymbol{x}-\boldsymbol{k}\right), 0 \leqslant \boldsymbol{k}<2^{n}\right\} .
$$

For $\Omega=(0,1)^{d}$, the exceptions related with the boundary are found in the spaces $W_{n}$ with a zero index (i.e., $\|\boldsymbol{n}\|=0$ ). These $W_{n}$ have basis functions with different shapes. They are derived from the corresponding functions for the unbounded case, but their support is restricted to $\Omega$. Their corresponding nodal points $z_{n-e, k}$, are found on the boundary $\partial \Omega=\bar{\Omega} \backslash \Omega$ instead of in the interior. For $\|\boldsymbol{n}\|=0$ we have $W_{n}$ spanned by a basis

$$
\left\{\begin{array}{l|l}
\psi_{\boldsymbol{n}, \boldsymbol{k}}(\boldsymbol{x}) \mid \begin{array}{ll}
0 \leqslant k_{j}<2^{n_{j}-1}, & \text { if } n_{j} \neq 0, \\
k_{j}=0, & \text { if } n_{j}=0,
\end{array} \quad j=1, \ldots, d
\end{array}\right\} .
$$

Taking such modifications into account, both for $\Omega=(0,1)^{d}$ and for $\Omega=\mathbb{R}^{d}$, for each $u \in L_{\text {loc }}^{p}(\Omega)$ we may write a hierarchical expansion (a wavelet expansion) according to (38) or (51), as

$$
u(\boldsymbol{x})=\sum_{\boldsymbol{n} \in \mathbb{Z}} w_{\boldsymbol{n}}=\sum_{\boldsymbol{n}, \boldsymbol{k}} c_{\boldsymbol{n} \boldsymbol{k}} \psi_{\boldsymbol{n}, \boldsymbol{k}}(\boldsymbol{x})=\sum_{\boldsymbol{n}, \boldsymbol{k}} c_{\boldsymbol{n} \boldsymbol{k}} \widehat{\psi}\left(2^{n} \boldsymbol{x}-\boldsymbol{k}\right),
$$

where $\widehat{\psi}$ is simply

$$
\widehat{\psi}(\boldsymbol{x})= \begin{cases}\operatorname{sign}\|\boldsymbol{x}\|, & \text { if } \max \left(x_{1}, \ldots, x_{d}\right)<1 \\ 0, & \text { if } \max \left(x_{1}, \ldots, x_{d}\right)>1\end{cases}
$$

and $c_{\boldsymbol{n} \boldsymbol{k}}=0$ for all $\boldsymbol{k}$ with $\|\boldsymbol{k}\|$ even.

\subsubsection{Piecewise d-linear approximation}

We approximate $u \in X=C^{0}(\Omega)$ by $u_{n} \in V_{n}$, in the space of piecewise $d$-linear functions on $\Omega_{n}$, i.e., in

$$
V_{\boldsymbol{n}}=\operatorname{Span}\left(\left\{\phi_{\boldsymbol{n} \boldsymbol{j}}\right\}\right)
$$

with, for some $q \geqslant 1$ or $q=\infty$,

$$
\begin{aligned}
& \phi_{\boldsymbol{n} \boldsymbol{j}}(\boldsymbol{x})=2^{|\boldsymbol{n}| / q} \phi\left(2^{n} \boldsymbol{x}-\boldsymbol{j}\right) \\
& \phi(\boldsymbol{x})=\prod_{j=1}^{d} \Lambda\left(x_{j}\right), \quad \text { with } \Lambda(x)=\max (0,1-|x|) \text { the usual hat function. }
\end{aligned}
$$

Clearly this is a basis for a tensor product space as (46), where $V_{n_{j}}$ are spaces of piecewise linear functions on a partitioning of $\Omega_{j}$ with meshwidth $h_{j}=2^{-n_{j}}$. The set of nodal points $\left\{j 2^{n}\right\}_{j \in \mathbb{Z}^{\prime}}$ in
$\Omega$ is denoted by $\Omega_{n}^{+}$.

Here we define the restriction $R_{n}$ as the projection

$$
\begin{aligned}
R_{n}: X & \rightarrow V_{n} \subset X, \\
u & \mapsto u_{n}=R_{n} u, \quad u_{n}(\boldsymbol{x})=u(\boldsymbol{x}) \forall \boldsymbol{x} \in \Omega_{n}^{+} .
\end{aligned}
$$


The restriction is also of type (30) and the operator $R_{n}=R_{n_{1}, \ldots, n_{d}}$ can be decomposed as (48) where $R_{n_{j} \boldsymbol{e}_{j}} u(\boldsymbol{x})$ is a function, piecewise linear in the $j$ th coordinate direction, on a partitioning $\Omega_{n}$, such that $R_{n_{j} \boldsymbol{e}_{j}} u(\boldsymbol{x})=u(\boldsymbol{x})$ for all $\boldsymbol{x}$ with $x_{j} / h_{j} \in \mathbb{Z}$.

It is clear that there exists a basis-function in $V_{n}$ for each nodal point $\boldsymbol{x}_{n k}$ in $\bar{\Omega}_{n}^{+}$. If and only iff $\|\boldsymbol{k}\|$ is even, there exists a 'parent grid' $\bar{\Omega}_{m}^{+}$with $\boldsymbol{m} \leqslant \boldsymbol{n}$ and $\boldsymbol{m} \neq \boldsymbol{n}$, for which $x_{n k} \in \bar{\Omega}_{m}^{+}$. Hence, in this case each wavelet space $W_{n}$ has its natural basis

$$
\left\{\phi_{n k} \mid k \in \mathbb{Z}^{d} \text { with }\|\boldsymbol{k}\| \text { odd }\right\} \text {. }
$$

On the closed cube $\bar{\Omega}=[0,1]^{d}$ we consider the $\prod_{j=1}^{d}\left(2^{n_{j}}+1\right)$-dimensional spaces $V_{n}=V_{n}(\Omega)=$ $\bigotimes_{j=1}^{d} V_{n_{j}}([0,1])$. With homogeneous Dirichlet boundary conditions, the dimension of the corresponding space $V_{n}^{0} \subset V_{n}$ is $\prod_{j=1}^{d}\left(2^{n_{j}}-1\right)$. It is immediately clear that typical FE-basis functions for $V_{\boldsymbol{n}}$ are the $d$-dimensional hat-functions: functions that vanish on all but one point of $\bar{\Omega}_{n}^{+}$. Each such FE basis function of $V_{n}^{0}$ is characterized by an interior point from $\Omega_{n}^{+}$.

We notice that for $\bar{\Omega}=[0,1]^{d}$ we have

$$
\begin{array}{ll}
V_{\boldsymbol{n}}=\{\boldsymbol{0}\} & \text { except for } \boldsymbol{n} \geqslant \mathbf{0}, \\
V_{\boldsymbol{n}}^{0}=\{\boldsymbol{0}\} & \text { except for } \boldsymbol{n} \geqslant \boldsymbol{e} .
\end{array}
$$

With $W_{n}$ (or $W_{n}^{0}$ ) we denote the subspace of $V_{n}$ (respectively $V_{n}^{0}$ ) of functions that vanish at the gridpoints of all $\bar{\Omega}_{n-e_{j}}^{+}$(respectively $\Omega_{n-e_{j}}^{+}$), j=1, .,d. From (55) we see that for $\Omega=\mathbb{R}^{d}$

$$
W_{n}=\operatorname{Span}\left(\left\{\phi_{n j} \mid\|j\| \text { odd }\right\}\right)
$$

and for $\Omega=[0,1]^{d}$ we see that $0 \leqslant j \leqslant 2^{n}$ and

$$
W_{\boldsymbol{n}}=\operatorname{Span}\left(\left\{\begin{array}{l|l}
\left.\phi_{\boldsymbol{n} j} \mid \begin{array}{l}
j_{i} \text { odd, } 0<j_{i}<2^{n_{i}}, \\
\text { if } n_{i}>0, \\
j_{i}=0,1,
\end{array} \quad i=1, \ldots, d\right\}
\end{array}\right\}\right)
$$

Clearly $W_{\boldsymbol{n}}=W_{\boldsymbol{n}}^{0}=\{0\}$, except for $\boldsymbol{n} \geqslant \mathbf{0}$. If $\|\boldsymbol{n}\|=0$ we see that $V_{\boldsymbol{n}}^{0}=\{0\}$ and $W_{\boldsymbol{n}}$ is spanned by FE basis functions that are characterized by boundary points on the unit cube. Thus, the trace of a function on the boundary is exclusively approximated by elements of $W_{n}$ with $\|\boldsymbol{n}\|=0$. Further we see $W_{n}^{0}=W_{n}$ if $\boldsymbol{n} \geqslant \boldsymbol{e}$. Apparently

$$
V_{n}=\bigoplus_{0 \leqslant k \leqslant n} W_{k}, \quad \text { and } \quad V_{n}^{0}=\bigoplus_{e \leqslant k \leqslant n} W_{k}
$$

\subsection{Error estimates for regular grids}

The decompositions of type (38) allow the approximation of a sufficiently smooth function in $X(\Omega)$ by a series with elements in $W_{j}$. To obtain an impression of the quality of these expansions in the following sections we derive error estimates. 


\subsubsection{Estimates for piecewise constant approximation}

As the case where domain boundaries are present, is the more general one, we study the case $\Omega=(0,1)^{d}$. To quantify the error of approximation on $\Omega$, we introduce for $u \in C^{e}(\bar{\Omega})$ the seminorm

$$
|u|=\max _{\boldsymbol{x} \in \Omega}\left|D^{\boldsymbol{e}} u(\boldsymbol{x})\right|+\max _{\substack{\boldsymbol{0} \leqslant \boldsymbol{p} \leqslant \boldsymbol{e} \\ 0<|\boldsymbol{p}|<d}} \max _{\boldsymbol{x} \in \bar{\Omega} \backslash \Omega}\left|D^{\boldsymbol{p}} u(\boldsymbol{x})\right| .
$$

Now we derive the following

Theorem 3.1. If we consider an expansion of $a C^{e}(\bar{\Omega})$-function, $u$, in piecewise constant functions on the grid $\Omega_{\boldsymbol{n}}$, for an arbitrary $\boldsymbol{n} \in \mathbb{Z}^{d}, \boldsymbol{n}>\mathbf{0}$, and if we write

$$
R_{\boldsymbol{n}} u=\sum_{\mathbf{0} \leqslant \boldsymbol{m} \leqslant \boldsymbol{n}} w_{\boldsymbol{m}}
$$

with $w_{\boldsymbol{m}} \in W_{\boldsymbol{m}}, \mathbf{0} \leqslant \boldsymbol{m} \leqslant \boldsymbol{n}$, then, for $\boldsymbol{m} \neq \mathbf{0}$ we have

$$
\left\|w_{m}\right\|_{L_{2}(\Omega)} \leqslant 2^{-d / 2} 2^{-|m|}|u|,
$$

and an estimate for the approximation error

$$
\left\|u-R_{\boldsymbol{n}} u\right\|_{L_{2}(\Omega)} \leqslant(2 / 3)^{d / 2}\left\|\boldsymbol{h}_{\boldsymbol{n}}\right\||u| .
$$

Proof. We take $\left\{\psi_{m \boldsymbol{k}}\right\}$ as a basis in $W_{\boldsymbol{m}}, \boldsymbol{e} \leqslant \boldsymbol{m} \leqslant \boldsymbol{n}$. All these functions form an $L_{2}(\Omega)$-orthonormal set (orthonormal Haar basis) and they are orthogonal to all functions in $W_{n}, \boldsymbol{n} \neq \boldsymbol{m}$.

Thus, we find (65) with $w_{m}=\sum_{k} a_{m k} \psi_{m k}$, where

$$
a_{\boldsymbol{m} \boldsymbol{k}}=\left(u, \psi_{\boldsymbol{m}, \boldsymbol{k}}\right)=\int_{\Omega} u \psi_{\boldsymbol{m}, \boldsymbol{k}} \mathrm{d} \Omega=\int_{\Omega_{\boldsymbol{m}-\boldsymbol{e}, \boldsymbol{k}}} u \psi_{\boldsymbol{m}, \boldsymbol{k}} \mathrm{d} \Omega .
$$

For $\boldsymbol{m} \geqslant \boldsymbol{e}$ the point $\boldsymbol{z}_{\boldsymbol{m}-\boldsymbol{e}, \boldsymbol{k}}$ lies in the interior of $\Omega$ and the estimate holds with

$$
|u|=\max _{\boldsymbol{x}}\left|D^{e} u(\boldsymbol{x})\right| .
$$

Viz., by Taylor expansion around $z_{m-e, k}$, we have

$$
\begin{aligned}
\left|a_{\boldsymbol{m} \boldsymbol{k}}\right| & =\left|\int_{\Omega_{\boldsymbol{m}-\boldsymbol{e}, \boldsymbol{k}}} u \psi_{\boldsymbol{m}, \boldsymbol{k}} \mathrm{d} \Omega\right| \\
& \leqslant\left|\int_{\Omega_{\boldsymbol{m}-\boldsymbol{e}, \boldsymbol{k}}}\left\|\boldsymbol{x}-\boldsymbol{z}_{\boldsymbol{m}-\boldsymbol{e}, \boldsymbol{k}}\right\|\right| u\left|\psi_{\boldsymbol{m}, \boldsymbol{k}} \mathrm{d} \Omega\right| \\
& =|u| 2^{|\boldsymbol{m}-\boldsymbol{e}| / 2} 2^{d} \prod_{j=1}^{d} \int_{0}^{2^{-m_{j}}} \xi_{j} \mathrm{~d} \xi_{j} \\
& =|u| 2^{-d / 2} 2^{-3|\boldsymbol{m}| / 2} .
\end{aligned}
$$

For $\boldsymbol{m} \neq \mathbf{0}, \boldsymbol{m} \ngtr e$, i.e., for $\psi_{m k}$ with an $\boldsymbol{m}$-component equal to zero, the point $\boldsymbol{z}_{m-e, k}$ lies on the boundary and the function $\psi_{m, k}$ is constant in one direction over the whole domain $\Omega$, and it is of 
Haar-wavelet type for the non-zero indices (or index). In this case the orthonormal basis functions are $2^{\beta / 2} \psi_{m, k}$, where $\beta$ is the number of zeroes in $\boldsymbol{m}$. Nevertheless, provided that $\boldsymbol{m} \neq \mathbf{0}$, in this situation the same estimate (68) holds with, e.g., if $m_{1}=0$ and $m_{j} \geqslant 1$ for $j=2, \ldots, d$,

$$
|u|=\max _{\boldsymbol{x}}\left|\frac{\partial^{d-1} u(\boldsymbol{x})}{\partial x_{2} \cdots \partial x_{d}}\right|
$$

Hence, the estimate (68) holds for $\boldsymbol{m} \geqslant \mathbf{0}, \boldsymbol{m} \neq \mathbf{0}$, if we use the seminorm (64), and we find

$$
\left\|w_{m}\right\|^{2}=\sum_{k}\left|a_{m k}\right|^{2} \leqslant \sum_{k} 2^{-d} 2^{-3|m|}|u|^{2}=2^{-d} 2^{-2|m|}|u|^{2},
$$

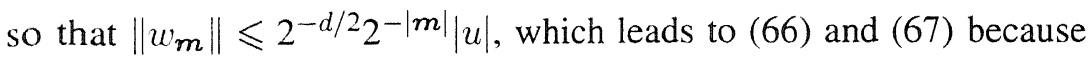

$$
\begin{aligned}
\left\|u-R_{\boldsymbol{n}} u\right\|^{2} & =\sum_{\substack{m_{1}>n_{1} \\
\text { or } \\
m_{d}>n_{d}}}\left\|w_{\boldsymbol{m}}\right\|^{2} \leqslant \sum_{j=1}^{d} \sum_{\substack{m_{j} \geqslant \mathbf{0} \\
m_{j}>n_{j}}} 2^{-d} 2^{-2|\boldsymbol{m}|}|u|^{2} \\
& \leqslant 2^{-d}|u|^{2} \sum_{j=1}^{d} \sum_{\substack{m \geqslant 0 \\
m_{j}>n_{j}}}(1 / 4)^{m_{1}+\cdots+m_{d}} \\
& \leqslant|u|^{2}(2 / 3)^{d} \sum_{j=1}^{d} h_{n_{j}}^{2} \leqslant|u|^{2}(2 / 3)^{d}\left\|\boldsymbol{h}_{\boldsymbol{n}}\right\|^{2} .
\end{aligned}
$$

If we have no further a priori knowledge about $u$, the most efficient approximation will be one with $h_{1}=\cdots=h_{d}$ because this equalizes the main terms in the error bound. We see that the truncation error for $u-R_{n} u$ is not particularly promising or surprising: the major part of the error is produced by the largest meshwidth: $\left(\max \left(h_{1}, \ldots, h_{d}\right)\right)^{d / 2}$, whereas the total number of degrees of freedom for an element in $V_{n}$ is $2^{|n|}$.

\subsubsection{Estimates for piecewise d-linear approximation}

For a function $u \in C^{\mathbf{0}}(\Omega)$ we consider piecewise linear approximation as in Section 3.1.2. We approximate $u$ by $u_{n} \in V_{n}$, where $V_{n}$ is the space of piecewise $d$-linear functions on $\Omega_{n}$. We take $u_{n}$ such that $u_{n}(\boldsymbol{x})=u(\boldsymbol{x})$ for all $x \in \Omega_{n}^{+}$and we write

$$
u_{\boldsymbol{n}}(\boldsymbol{x})=\sum_{\boldsymbol{j}} d_{\boldsymbol{n} \boldsymbol{j}} \phi_{\boldsymbol{n} \boldsymbol{j}}(\boldsymbol{x}),
$$

where $\phi_{\boldsymbol{n} j}(\boldsymbol{x})$ is defined by (56).

With $u_{n} \in V_{n}$ the piecewise linear approximation on $\Omega_{n}$ of the function $u \in C^{0}(\Omega)$, we make the hierarchical decomposition $V_{n}=\bigoplus_{k \leqslant n} W_{k}$, and write

$$
u_{n}=\sum_{k \leqslant n} w_{k}, \quad w_{k} \in W_{k},
$$

where

$$
w_{k}(\boldsymbol{x})=\sum_{j} c_{k j} \phi_{k j}(\boldsymbol{x}),
$$


with $c_{k j}=0$ for all $j$ with $\|\boldsymbol{j}\|$ even.

In practice the coefficients $c_{k j},\|j\|$ odd, are computed as hierarchical surplus coefficients, by taking the difference between the value $u\left(j h_{k}\right)$ and the interpolant from coarser grids. This is most conveniently formulated by introducing stencil notation. Therefore, we introduce the difference operator

$$
\nabla_{\boldsymbol{h}} u(\boldsymbol{z})=u(\boldsymbol{z}+\boldsymbol{h})-u(\boldsymbol{z})
$$

and the usual central difference approximation for the second derivative by stencil notation, as

$$
\left[\frac{1}{2},-1, \frac{1}{2}\right]_{h_{j} e_{j}} u(z)=\frac{1}{2} \nabla_{h_{j} e_{j}}^{2} u\left(z-h_{j} e_{j}\right) .
$$

With this notation we write an expression for the hierarchical coefficients in a piecewise linear approximation. We see that $d$-linear interpolation leads to the following expression for the hierarchical
surplus coefficient

$$
c_{\boldsymbol{k} j}\left\|\boldsymbol{h}_{\boldsymbol{k}}\right\|^{-1 / q}=\prod_{j=1, d}\left[-\frac{1}{2}, 1,-\frac{1}{2}\right]_{h_{j} \boldsymbol{e}_{j}} u\left(\boldsymbol{j} \boldsymbol{h}_{\boldsymbol{k}}\right) .
$$
Notice that the factor $\left\|\boldsymbol{h}_{\boldsymbol{k}}\right\|^{1 / q}$ cancels the scaling factor $2^{|\boldsymbol{k}| / q}$ in the definition of $\phi_{\boldsymbol{k} j}$, so that the
function $u(\boldsymbol{x})$ is expanded as

$$
u(\boldsymbol{x}) \approx \sum_{\boldsymbol{k}, \boldsymbol{j}} c_{\boldsymbol{k} j}\left\|\boldsymbol{h}_{\boldsymbol{k}}\right\|^{-1 / q} \phi\left(2^{\boldsymbol{k}} \boldsymbol{x}-\boldsymbol{j}\right) .
$$

An expression for the coefficient $c_{k j}$ is found in the following lemma.

Lemma 3.2. Let $u \in C^{\boldsymbol{e}+\boldsymbol{m}}$, for a given $\boldsymbol{m}$ with $\mathbf{0} \leqslant \boldsymbol{m} \leqslant \boldsymbol{e}$, and let

$$
L_{\boldsymbol{n} \boldsymbol{j}}(\boldsymbol{x})=2^{-|\boldsymbol{n}|} \phi\left(2^{n} \boldsymbol{x}-\boldsymbol{j}\right)
$$

then, for each $\phi_{n j} \in W_{n},\|\boldsymbol{n}\| \neq 0,\|\boldsymbol{j}\|$ odd, we have

$$
\begin{aligned}
\left\|\boldsymbol{h}_{\boldsymbol{n}}\right\|^{-1 / q}\left|c_{\boldsymbol{n} \boldsymbol{j}}\right| & =\prod_{i=1}^{d}\left[-\frac{1}{2}, 1,-\frac{1}{2}\right]_{h_{i} \boldsymbol{e}_{i}} u\left(\boldsymbol{j} \boldsymbol{h}_{\boldsymbol{n}}\right) \\
& =(-1)^{|\boldsymbol{e}+\boldsymbol{m}|} 2^{-d} \int_{\Omega} D^{\boldsymbol{e}+\boldsymbol{m}} u(\boldsymbol{x}) D^{\boldsymbol{e}-m} L_{\boldsymbol{n} \boldsymbol{j}}(\boldsymbol{x}) \mathrm{d} \Omega .
\end{aligned}
$$

Proof. We see that for $\|\boldsymbol{n}\| \neq 0,\|\boldsymbol{j}\|$ odd, each $L_{\boldsymbol{n} \boldsymbol{j}}$ has a support in the interior of $\Omega$. Taking this into account, we give the proof after a coordinate translation with $2^{m} j$ then we see that for all
$i, \quad 0 \leqslant i \leqslant d$,

$$
\sum_{s_{i}=-1,1} \int_{0}^{s_{i} h_{i}} D^{\boldsymbol{e}_{i}} u(\boldsymbol{z}) \mathrm{d} z_{i}=\left.\sum_{s_{i}=-1,1} u(\boldsymbol{z})\right|_{z_{i}=0} ^{s_{i} h_{i}}=\left.\sum_{s_{i}=-1,1} \nabla_{s_{i} h_{i} \boldsymbol{e}_{i}} u(\boldsymbol{z})\right|_{z_{i}=0}=\left.[1,-2,1]_{h_{i} \boldsymbol{e}_{i}} u(\boldsymbol{z})\right|_{z_{i}=0}
$$

and hence 


$$
\begin{aligned}
& \prod_{i=1, d}\left[-\frac{1}{2}, 1,-\frac{1}{2}\right]_{h_{i} \boldsymbol{e}_{i}} u(\mathbf{0}) \\
& =\left(-\frac{1}{2}\right)^{d} \prod_{i=1, d}\left[\sum_{s_{i}=-1,1} \int_{0}^{s_{i} h_{i}} D^{\boldsymbol{e}_{i}} \cdot \mathrm{d} x_{i}\right] u(x) \\
& =\left(-\frac{1}{2}\right)^{d} \sum_{s_{1}, \ldots, s_{d}=-1,1} \int_{0}^{s_{1} h_{1}} \cdots \int_{0}^{s_{d} h_{d}} D^{\boldsymbol{e}} u(\boldsymbol{x}) \mathrm{d} x_{1} \cdots \mathrm{d} x_{d} \\
& =\left(-\frac{1}{2}\right)^{d} \int_{-h_{1}}^{h_{1}} \ldots \int_{-h_{d}}^{h_{d}} D^{\boldsymbol{e}} u(\boldsymbol{x}) \prod_{i=1}^{d}\left(2^{-n_{i}} \frac{\mathrm{d}}{\mathrm{d} x_{i}} \Lambda\left(2^{n_{i}} x_{i}\right)\right) \mathrm{d} x_{1} \cdots \mathrm{d} x_{d} \\
& =\left(-\frac{1}{2}\right)^{d} \int^{\boldsymbol{e}} u(\boldsymbol{x}) 2^{-|\boldsymbol{n}|} D^{\boldsymbol{e} \phi}\left(2^{|\boldsymbol{n}|} \boldsymbol{x}\right) \mathrm{d} \Omega \\
& =(-1)^{|\boldsymbol{e}+\boldsymbol{m}|} 2^{-d} \int^{\boldsymbol{e}+\boldsymbol{m}} u(\boldsymbol{x}) D^{\boldsymbol{e}-\boldsymbol{m}} L_{\boldsymbol{n} \mathbf{0}}(\boldsymbol{x}) \mathrm{d} \Omega
\end{aligned}
$$

\section{Remarks.}

(1) For $\|\boldsymbol{n}\|=0$ (i.e., for boundary points), the same formula holds, provided that the formula is restricted to the lower dimensional boundary manifold (e.g., the face or the edge of the unit cube).

(2) For an $\boldsymbol{m}$ with $\mathbf{0} \leqslant \boldsymbol{m} \leqslant \boldsymbol{e}$ we derive an expression for $\left\|D^{\boldsymbol{m}} \phi\right\|_{p}$, with $\phi$ given by (56) as follows:

$$
\left\|D^{\boldsymbol{m}} \phi\right\|_{p}^{p}=\int_{\Omega} \prod_{i}\left|D^{m_{i}} \Lambda\left(x_{i}\right)\right|^{p} \mathrm{~d} \Omega=2^{d}(p+1)^{-|\boldsymbol{e}-\boldsymbol{m}|} .
$$

So that

$$
\left\|D^{\boldsymbol{m}} \phi\right\|_{p}=2^{d / p}(p+1)^{-|e-m| / p} .
$$

(3) In (56) we have $\phi_{n j}=2^{|n| / q} \phi\left(2^{n} x-j\right)$, and hence

$$
\left\|D^{\boldsymbol{m}} \phi_{n j}\right\|_{p}^{p}=\int 2^{|\boldsymbol{n}| p / q}\left|D^{\boldsymbol{m}} \phi\left(2^{n} \boldsymbol{x}-j\right)\right|^{p} \mathrm{~d} \Omega=2^{|\boldsymbol{n}|(p / q-1)} 2^{|\boldsymbol{m} \circ \boldsymbol{n}| p}\left\|D^{\boldsymbol{m}} \phi\right\|_{p}^{p} .
$$

Here,

$$
2^{\boldsymbol{m} \circ \boldsymbol{n}}=\prod_{i=1}^{d} 2^{m_{i} n_{i}}=\prod_{i=1}^{d} h_{n_{i}}^{-m_{i}}=\boldsymbol{h}_{n}^{-\boldsymbol{m}}
$$

so that for arbitrary $j$,

$$
\left\|D^{\boldsymbol{m}} \phi_{\boldsymbol{n} j}\right\|_{p}=\left\|\boldsymbol{h}_{\boldsymbol{n}}\right\|^{(1 / p-1 / q)} \boldsymbol{h}_{\boldsymbol{n}}^{-\boldsymbol{m}}\left\|D^{\boldsymbol{m}} \phi\right\|_{p} .
$$

This means that the norm $\left\|\phi_{n j}\right\|_{p}$ is independent of the level $\boldsymbol{n}$ iff we take $q=p$. 
(4) To obtain error estimates in the approximation Theorem 3.3 we compute an expression for $\left\|D^{\boldsymbol{m}} L_{\boldsymbol{n} j}(\boldsymbol{x})\right\|_{p}$, with $L_{\boldsymbol{n} j}$ as introduced in (75), in particular for $p=1,2, \infty$.

$$
\left\|D^{\boldsymbol{m}} L_{\boldsymbol{n} \boldsymbol{j}}(\boldsymbol{x})\right\|_{p}^{p}=\left\|\boldsymbol{h}_{\boldsymbol{n}}\right\|^{p+1} \boldsymbol{h}_{\boldsymbol{n}}^{-p \boldsymbol{m}}\left\|D^{\boldsymbol{m}} \phi\right\|_{p}^{p}=\left\|\boldsymbol{h}_{\boldsymbol{n}}\right\|^{p+1} \boldsymbol{h}_{\boldsymbol{n}}^{-p \boldsymbol{m}} 2^{d}(p+1)^{-|\boldsymbol{e}-\boldsymbol{m}|} .
$$

So that we may conclude, also considering the special case $p=\infty$,

$$
\begin{aligned}
& \left\|D^{m} L_{\boldsymbol{n} j}(\boldsymbol{x})\right\|_{1}=\left\|\boldsymbol{h}_{\boldsymbol{n}}\right\|^{2}\left(\boldsymbol{h}_{\boldsymbol{n}} / 2\right)^{-\boldsymbol{m}}, \\
& \left\|D^{m} L_{\boldsymbol{n}}(\boldsymbol{x})\right\|_{2}=(2 / 3)^{d / 2}\left\|\boldsymbol{h}_{\boldsymbol{n}}\right\|^{3 / 2}\left(\boldsymbol{h}_{\boldsymbol{n}} / \sqrt{3}\right)^{-\boldsymbol{m}}, \\
& \left\|D^{m} L_{\boldsymbol{n}}(\boldsymbol{x})\right\|_{\infty}=\left\|\boldsymbol{h}_{\boldsymbol{n}}\right\|\left(\boldsymbol{h}_{\boldsymbol{n}}\right)^{-\boldsymbol{m}} .
\end{aligned}
$$

Using the above expressions and Lemma 3.2, we can derive the error estimates in the following theorem.

Theorem 3.3. Let $u \in C_{0}^{e+m}(\Omega)$ be given for some $\boldsymbol{m}$ with $\mathbf{0} \leqslant \boldsymbol{m} \leqslant \boldsymbol{e}$, and let $u_{n} \in V_{n}$ be the piecewise linear approximation on $\Omega_{n}$ of $u$, such that $u_{\boldsymbol{n}}(\boldsymbol{x})=u(\boldsymbol{x})$ for all $\boldsymbol{x} \in \Omega_{\boldsymbol{n}}^{+}$. If we make the hierarchical decomposition $V_{n}=\bigoplus_{k \leqslant n} W_{k}$, and write

$$
u_{\boldsymbol{n}}=\sum_{\boldsymbol{k} \leqslant n} w_{\boldsymbol{k}}, \quad w_{\boldsymbol{k}} \in W_{\boldsymbol{k}}
$$

then we have the estimates

$$
\begin{aligned}
& \left\|w_{\boldsymbol{k}}\right\|_{2} \leqslant\left\|D^{\boldsymbol{e}+\boldsymbol{m}} u\right\|_{2}\left\|\boldsymbol{h}_{\boldsymbol{k}}\right\|^{2} 2^{-d / 2} 3^{-|\boldsymbol{m}| / 2} \boldsymbol{h}_{\boldsymbol{k}}^{-(\boldsymbol{e}-\boldsymbol{m})} \\
& \left\|w_{\boldsymbol{k}}\right\|_{\infty} \leqslant\left\|D^{\boldsymbol{e}+\boldsymbol{m}} u\right\|_{\infty}\left\|\boldsymbol{h}_{\boldsymbol{k}}\right\|^{2}\left(\boldsymbol{h}_{\boldsymbol{k}} / 2\right)^{-(\boldsymbol{e}-\boldsymbol{m})} \\
& \left\|u-u_{\boldsymbol{n}}\right\|_{2} \leqslant\left\|D^{\boldsymbol{e}+\boldsymbol{m}} u\right\|_{2} 2^{-d} 3^{-3|\boldsymbol{m}| / 2} \sum_{i=1}^{d} h_{n_{i}}^{\left(1+m_{i}\right)} \\
& \left\|u-u_{\boldsymbol{n}}\right\|_{\infty} \leqslant\left\|D^{\boldsymbol{e}+\boldsymbol{m}} u\right\|_{\infty} 6^{-|\boldsymbol{m}|} \sum_{i=1}^{d} h_{n_{i}}^{\left(1+m_{i}\right)}
\end{aligned}
$$

Proof. Using (74) and Lemma 3.2 we can obtain estimates for the hierarchical coefficients $c_{\boldsymbol{k} j}$. We fix $k$ and we derive, writing $h=h_{k}$,

$$
\begin{aligned}
\|\boldsymbol{h}\|^{-1 / q}\left|c_{\boldsymbol{k} \boldsymbol{j}}\right| & =2^{-d}\left|\int_{\Omega} D^{\boldsymbol{e}+\boldsymbol{m}} u(\boldsymbol{x}) \chi_{\boldsymbol{k} \boldsymbol{j}}(\boldsymbol{x}) D^{\boldsymbol{e}-\boldsymbol{m}} L_{\boldsymbol{k} \boldsymbol{j}}(\boldsymbol{x}) \mathrm{d} \boldsymbol{x}\right| \\
& \leqslant 2^{-d}\left\|D^{\boldsymbol{e}+\boldsymbol{m}} u \chi_{\boldsymbol{k} \boldsymbol{j}}\right\|_{\infty}\left\|D^{\boldsymbol{e}-\boldsymbol{m}} L_{\boldsymbol{k} \boldsymbol{j}}\right\|_{1} \\
& \leqslant 2^{-d}\left\|D^{\boldsymbol{e}+\boldsymbol{m}} u \chi_{\boldsymbol{k} j}\right\|_{\infty}\|\boldsymbol{h}\|^{2}(\boldsymbol{h} / 2)^{-(\boldsymbol{e}-\boldsymbol{m})},
\end{aligned}
$$

where $\chi_{\boldsymbol{k} j}$ is the characteristic function for the support of $L_{\boldsymbol{k} \boldsymbol{j}}(\boldsymbol{x})$, or similarly

$$
\begin{aligned}
\|\boldsymbol{h}\|^{-1 / q}\left|c_{\boldsymbol{k} \boldsymbol{j}}\right| & =2^{-d}\left|\int_{\Omega} D^{\boldsymbol{e + m}} u(\boldsymbol{x}) \chi_{\boldsymbol{k} j} D^{\boldsymbol{e}-\boldsymbol{m}} L_{\boldsymbol{k} \boldsymbol{j}}(\boldsymbol{x}) \mathrm{d} \boldsymbol{x}\right| \\
& \leqslant 2^{-d}\left\|D^{\boldsymbol{e}+\boldsymbol{m}} u \chi_{\boldsymbol{k} \boldsymbol{j}}\right\|_{2}\left\|D^{\boldsymbol{e}-\boldsymbol{m}} L_{\boldsymbol{k} \boldsymbol{j}}\right\|_{2} \\
& \leqslant 6^{-d / 2}\left\|D^{\boldsymbol{e}+\boldsymbol{m}} u \chi_{\boldsymbol{k} \boldsymbol{j}}\right\|_{2}\|\boldsymbol{h}\|^{3 / 2}(\boldsymbol{h} / \sqrt{3})^{-(\boldsymbol{e}-\boldsymbol{m})} .
\end{aligned}
$$


We write $w_{\boldsymbol{k}}==\sum_{\boldsymbol{j}} c_{\boldsymbol{k} \boldsymbol{j}} \phi_{\boldsymbol{k} \boldsymbol{j}}$ with $\|\boldsymbol{j}\|$ odd, and we know that these functions $\left\{\phi_{\boldsymbol{k} \boldsymbol{j}}\right\}_{\boldsymbol{j}}$, for fixed $\boldsymbol{k}$ have disjoint supports. Hence, for the hierarchical contribution,

$$
\begin{aligned}
\left\|w_{\boldsymbol{k}}\right\|_{2}^{2} & =\left\|\sum_{\boldsymbol{i}} c_{\boldsymbol{k} \boldsymbol{i}} \phi_{\boldsymbol{k} i}\right\|_{2}^{2}=\sum_{\boldsymbol{i}, \boldsymbol{j}} c_{\boldsymbol{k} \boldsymbol{i}} c_{\boldsymbol{k} \boldsymbol{j}} \int \phi_{\boldsymbol{k} \boldsymbol{i}} \phi_{\boldsymbol{k} j} \mathrm{~d} \Omega=\sum_{\boldsymbol{i}} c_{\boldsymbol{k} \boldsymbol{i}}^{2}\left\|\phi_{\boldsymbol{k} \boldsymbol{i}}\right\|_{2}^{2} \\
& \leqslant\|\boldsymbol{h}\|^{2 / q}\left\|\phi_{\boldsymbol{k} \boldsymbol{i}}\right\|_{2}^{2} \sum_{\boldsymbol{j}}\left\|D^{\boldsymbol{e}+\boldsymbol{m}} u \chi_{\boldsymbol{k} \boldsymbol{j}}\right\|_{2}^{2} 6^{-d}\|\boldsymbol{h}\|^{3}(\boldsymbol{h} / \sqrt{3})^{-2(\boldsymbol{e}-\boldsymbol{m})} \\
& \leqslant\|\boldsymbol{h}\|^{4} 2^{-d}\left\|D^{\boldsymbol{e}+\boldsymbol{m}} u\right\|_{2}^{2} \boldsymbol{h}^{-2(\boldsymbol{e}-\boldsymbol{m})} 3^{-|\boldsymbol{m}|} .
\end{aligned}
$$

For the other norm $\left\|w_{\boldsymbol{k}}\right\|_{\infty}$ we obtain similarly

$$
\begin{aligned}
\left\|w_{\boldsymbol{k}}\right\|_{\infty} & =\left\|\sum_{\boldsymbol{j}} c_{\boldsymbol{k} \boldsymbol{j}} \phi_{\boldsymbol{k} \boldsymbol{j}}\right\|_{\infty} \leqslant \max _{\boldsymbol{j}}\left|c_{\boldsymbol{k} \boldsymbol{j}}\right|\left\|\phi_{\boldsymbol{k} \boldsymbol{j}}\right\|_{\infty} \\
& \leqslant\|\boldsymbol{h}\|^{1 / q} \max _{\boldsymbol{j}} 2^{-d}\left\|D^{\boldsymbol{e}+\boldsymbol{m}} u \chi_{\boldsymbol{k} \boldsymbol{j}}\right\|_{\infty}\|\boldsymbol{h}\|^{2}(h / 2)^{-(\boldsymbol{e}-\boldsymbol{m})}\|\boldsymbol{h}\|^{-1 / q} \\
& \leqslant 2^{-d}\left\|D^{\boldsymbol{e}+\boldsymbol{m}} u\right\|_{\infty}\|\boldsymbol{h}\|^{2}(\boldsymbol{h} / 2)^{-(\boldsymbol{e}-\boldsymbol{m})}
\end{aligned}
$$

For the error, for $p=2$ or $p=\infty$, we get

$$
\begin{aligned}
\left\|u-u_{\boldsymbol{n}}\right\|_{p} & =\left\|\sum_{\boldsymbol{k}} w_{\boldsymbol{k}}-\sum_{\boldsymbol{k} \leqslant n} w_{\boldsymbol{k}}\right\|_{p} \leqslant \sum_{\boldsymbol{k} \nless \boldsymbol{n}}\left\|w_{\boldsymbol{k}}\right\|_{p} \\
& \leqslant C_{p}\left\|D^{\boldsymbol{e}+\boldsymbol{m}} u\right\|_{p} \sum_{\boldsymbol{k} \nless \boldsymbol{n}}\left\|\boldsymbol{h}_{\boldsymbol{k}}\right\|^{2} \boldsymbol{h}_{\boldsymbol{k}}^{-(\boldsymbol{e}-\boldsymbol{m})} \\
& =C_{p}\left\|D^{\boldsymbol{e}+\boldsymbol{m}} u\right\|_{p} \sum_{\boldsymbol{k} \nless \boldsymbol{n}} h_{\boldsymbol{k}}^{\boldsymbol{e}+\boldsymbol{m}},
\end{aligned}
$$

with $C_{2}=2^{-d / 2} 3^{-|m| / 2}$ or $C_{\infty}=2^{-d} 2^{|e-m|}$. This yields the above mentioned estimates, by taking into account that

$$
\begin{aligned}
\sum_{k \nless n} h_{k}^{e+m} & =\sum_{k} h_{k}^{e+m}-\sum_{k \leqslant n} h_{k}^{e+m} \\
& =\prod_{i=1}^{d} \frac{1}{1-\left(\frac{1}{2}\right)^{1+m_{i}}}\left[1-\prod_{i=1}^{d}\left(1-\left(\frac{1}{2}\right)^{\left(1+n_{i}\right)\left(1+m_{i}\right)}\right)\right] \\
& \leqslant 2^{d}\left(\frac{2}{3}\right)^{|m|}\left[1-\prod_{i=1}^{d}\left(1-2^{-\left(1+n_{i}\right)\left(1+m_{i}\right)}\right)\right]=3^{-|m|} \sum_{i=1}^{d} h_{n_{i}}^{\left(1+m_{i}\right)} .
\end{aligned}
$$

For $\boldsymbol{m}=\boldsymbol{e}$ this simply reads $\sum_{\boldsymbol{k} \nless \boldsymbol{n}}\left\|\boldsymbol{h}_{\boldsymbol{k}}\right\|^{2} \leqslant 3^{-d}\left\|\boldsymbol{h}_{\boldsymbol{n}}\right\|^{2}$.

Corollary 3.4. As a direct corollary we find

$$
\left\|u-u_{\boldsymbol{n}}\right\|_{2} \leqslant 54^{-d / 2}\left\|D^{2 \boldsymbol{e}} u\right\|_{2}\left\|\boldsymbol{h}_{\boldsymbol{n}}\right\|^{2}, \quad\left\|u-u_{\boldsymbol{n}}\right\|_{\infty} \leqslant 6^{-d}\left\|D^{2 \boldsymbol{e}} u\right\|_{\infty}\left\|\boldsymbol{h}_{\boldsymbol{n}}\right\|^{2},
$$

and, for $p=2$ or $p=\infty$, and $\mathbf{0} \leqslant \boldsymbol{m} \leqslant \boldsymbol{e}$, 


$$
\begin{aligned}
& \left\|w_{\boldsymbol{k}}\right\|_{p} \leqslant C\left\|D^{\boldsymbol{e}+\boldsymbol{m}} u\right\|_{p} \boldsymbol{h}_{\boldsymbol{k}}^{\boldsymbol{e}+\boldsymbol{m}}, \\
& \left\|u-u_{\boldsymbol{n}}\right\|_{p} \leqslant C\left\|D^{\boldsymbol{e}+\boldsymbol{m}} u\right\|_{p} \sum_{i=1}^{d} h_{n_{i}}^{1+m_{i}}, \quad\left\|u-u_{\boldsymbol{n}}\right\|_{p} \leqslant C\left\|D^{2 \boldsymbol{e}} u\right\|_{p}\left\|\boldsymbol{h}_{\boldsymbol{n}}\right\|^{2} .
\end{aligned}
$$

From (78) also follows a bound by a $W_{p}^{e, \ell}$-norm. We immediately see that, for $0 \leqslant \ell \leqslant d$,

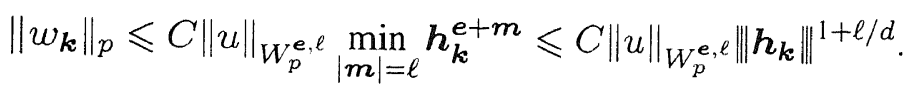

We gave the proof of Theorem 3.3 for functions that vanish at the boundary of $\Omega$. Taking into account the remark following Lemma 3.2, it is clear that similar estimates (with different constants and with terms including derivatives of $u$ that are restricted to the boundary planes as in (64)) also hold for functions with non-homogeneous boundary conditions.

\subsection{Error estimates for sparse grids}

\subsubsection{Estimates for piecewise constant approximation}

For piecewise constant approximation we use a sparse (box) grid $\Omega_{n}=\bigcup_{|\boldsymbol{k}| \leqslant n} \Omega_{\boldsymbol{k}} \cap \Omega$. A sparse grid approximation is obtained by interpolation on this grid by means of the space spanned by all $W_{k}$ with $|\boldsymbol{k}| \leqslant n$.

Theorem 3.5. Let $\widehat{R}_{n} u$ be the piecewise constant approximation of a function $u \in L_{2}(\Omega)$ on a sparse grid on level $n$ :

$$
\widehat{R}_{n} u=\sum_{|\boldsymbol{k}| \leqslant n} w_{k}, \quad w_{k} \in W_{k}
$$

then, with $\|\boldsymbol{h}\|=2^{-n}$, the volume of the finest cells, we have the estimate

$$
\left\|u-\widehat{R}_{n} u\right\|_{L_{2}(\Omega)} \leqslant C|u|\|\boldsymbol{h}\| \log (d-1) / 2\|\boldsymbol{h}\| .
$$

Proof. To prove the theorem for the $L_{2}(\Omega)$-norm, we use (69), and the orthogonality of the hierarchical basis functions, to obtain

$$
\left\|u-\widehat{R}_{n} u\right\|_{L_{2}(\Omega)}^{2} \leqslant \sum_{|\boldsymbol{k}|>n}\left\|w_{\boldsymbol{k}}\right\|_{L_{2}(\Omega)}^{2} \leqslant \sum_{|\boldsymbol{k}|>n} 2^{-d} 4^{-|\boldsymbol{k}|}|u|^{2}=2^{-d}|u|^{2} \sum_{|\boldsymbol{k}|>n} 2^{-2|\boldsymbol{k}|} .
$$

We know

$$
\sum_{l>n} 2^{-2 l}\left(\begin{array}{c}
l+d-1 \\
d-1
\end{array}\right)=2^{-2(n+1)}\left(\begin{array}{c}
n+d \\
d-1
\end{array}\right) F(1,1+n+d ; 2+n ; 1 / 4)=G(n, d) .
$$

Here $F$ is the hypergeometric function. It follows that

$$
G(n, d) \sim \frac{n^{d-1} 2^{-2 n}}{3(d-1) !} \quad \text { for } n \rightarrow \infty
$$


where the asymptotic value is reached soon for small values of $d$. Hence

$$
\left\|u-R_{n} u\right\|^{2} \leqslant 2^{-d}|u|^{2} \sum_{l>n} 2^{-2 l}\left(\begin{array}{c}
l+d-1 \\
d-1
\end{array}\right) \leqslant 2^{-d}|u|^{2} G(n, d) \leqslant 2^{-d}|u|^{2} C_{n d} \frac{n^{d-1} 2^{-2 n}}{3(d-1) !}
$$

where $C_{n d}$ is a constant that tends to one for large $n\left(C_{n d}<d\right.$ for $\left.n \geqslant 4\right)$. So, we conclude that

$$
\left\|u-R_{n} u\right\|_{L_{2}(\Omega)} \leqslant C|u| n^{(d-1) / 2} 2^{-n}
$$

which is equivalent with (82).

To guarantee a small error on a regular grid, in (67) all cell edges $h_{j}$ need to be small, but in (82) for the sparse grid only the volume $\|\boldsymbol{h}\|$ has to be small. Further, in the two-dimensional case, the estimate (82) is of a similar order of accuracy as (67), except for a logarithmic small factor. However, the number of degrees of freedom for the approximation (82) is significantly less. Namely, in the unit cube, for $R_{n} u$ the number of degrees of freedom is $2^{|n|}$, whereas for $\widehat{R}_{n} u$ it is $\mathrm{O}\left(n^{d-1} 2^{n}\right)$, viz. $2 n 2^{n}+1$ in the $2 \mathrm{D}$-case, and in the 3D-case, e.g., $\left(n^{2}+n+2\right) 2^{n}-1$. Because significantly less degrees of freedom are involved in the approximation $\widehat{R}_{n} u$ than in the approximation of $R_{(n, n, n)} u$, i.e., less coefficients $a_{j, k}$ and less gridpoints $z_{j, k}$, in analogy to [7], we call the approximation $\widehat{R}_{n} u$ the sparse grid approximation and

$$
\Omega_{n}^{\star}=\left\{\boldsymbol{z}_{j, \boldsymbol{k}}\left|\boldsymbol{z}_{\boldsymbol{j}, \boldsymbol{k}} \in Z_{\boldsymbol{n}},\right| \boldsymbol{n} \mid \leqslant n\right\}
$$

is the sparse (box) grid for this approximation on level $n$.

\subsubsection{Estimates for piecewise linear approximation}

For piecewise linear approximation we use a sparse (vertex) grid $\Omega_{n}^{+}=\bigcup_{|\boldsymbol{k}| \leqslant n} \Omega_{\boldsymbol{k}}^{+} \cap \Omega$. A sparse grid approximation is obtained by interpolation on this grid by means of the space spanned by all $W_{\boldsymbol{k}}$ with $|\boldsymbol{k}| \leqslant n$.

Theorem 3.6. Let $\widehat{R}_{n} u$ be the piecewise d-linear approximation of a function $u \in C_{0}^{e+m}(\Omega)$, with $\mathbf{0} \leqslant \boldsymbol{m} \leqslant \boldsymbol{e}$, on a sparse grid on level $n$ :

$$
\widehat{R}_{n} u=\sum_{|\boldsymbol{k}| \leqslant n} w_{\boldsymbol{k}}, \quad w_{\boldsymbol{k}} \in W_{\boldsymbol{k}}
$$

then, with $\|\boldsymbol{h}\|=2^{-n}$, the volume of the finest cells, we have for $p=2, \infty$, with $\boldsymbol{m}=\boldsymbol{e}$ the estimates

$$
\left\|u-\widehat{R}_{n} u\right\|_{p} \leqslant C\left\|D^{2 \boldsymbol{e}} u\right\|_{p}\|\boldsymbol{h}\|^{2} \log ^{d-1}\|\boldsymbol{h}\|^{-1},
$$

and with $|\boldsymbol{m}|<d$ the estimates

$$
\left\|u-\widehat{R}_{n} u\right\|_{p} \leqslant C\left\|D^{e+m} u\right\|_{p}\|\boldsymbol{h}\| \log ^{d-1-|\boldsymbol{m}|}\|\boldsymbol{h}\|^{-1},
$$

and with $0 \leqslant \ell \leqslant d$

$$
\left\|u-\widehat{R}_{n} u\right\|_{p} \leqslant C\|u\|_{W_{p}^{e, \ell}}\|\boldsymbol{h}\|^{1+\ell / d} \log ^{d-1}\|\boldsymbol{h}\|^{-1} .
$$

Proof. Using the estimates for $\left\|w_{k}\right\|_{p}$ from Theorem 3.3, we prove, more generally, for some $\boldsymbol{m}$ with $0 \leqslant m \leqslant e$, and for $p=2$ or $p=\infty$, 


$$
\begin{aligned}
\left\|u-\widehat{R}_{n} u\right\|_{p} & \leqslant \sum_{|\boldsymbol{k}|>n}\left\|w_{\boldsymbol{k}}\right\|_{p} \\
& \leqslant \sum_{|\boldsymbol{k}|>n} C\left\|D^{\boldsymbol{e}+\boldsymbol{m}} u\right\|_{p} \boldsymbol{h}_{\boldsymbol{k}}^{\boldsymbol{e}+\boldsymbol{m}} \\
& =C\left\|D^{\boldsymbol{e}+\boldsymbol{m}} u\right\|_{p} \sum_{|\boldsymbol{k}|>n} \boldsymbol{h}_{\boldsymbol{k}}^{\boldsymbol{e}+\boldsymbol{m}} \\
& \leqslant C\left\|D^{\boldsymbol{e}+\boldsymbol{m}} u\right\|_{p} 2^{-n}\left(C_{1} 2^{-n} n^{|\boldsymbol{m}|-1}+C_{2} n^{d-|\boldsymbol{m}|-1}\right)
\end{aligned}
$$

with $C_{1}=0$ if $|\boldsymbol{m}|=0$, and $C_{2}=0$ if $|\boldsymbol{m}|=d$. Hence, for $\boldsymbol{m} \neq \boldsymbol{e}$ we have

$$
\left\|u-\widehat{R}_{n} u\right\|_{p} \leqslant C\left\|D^{\boldsymbol{e}+\boldsymbol{m}} u\right\|_{p}\|\boldsymbol{h}\| \log ^{d-1-|m|}\|\boldsymbol{h}\|^{-1} .
$$

Moreover, (90) yields, for $\boldsymbol{m}=\boldsymbol{e}$,

$$
\left\|u-\widehat{R}_{n} u\right\|_{p} \leqslant C\left\|D^{2 \boldsymbol{e}} u\right\|_{p}\|\boldsymbol{h}\|^{2} \log ^{d-1}\|\boldsymbol{h}\|^{-1} .
$$

Further, using the estimate (80) we obtain, similar to the proof for Theorem 3.5,

$$
\begin{aligned}
& \left\|u-\widehat{R}_{n} u\right\|_{p} \leqslant \sum_{|\boldsymbol{k}|>n}\left\|w_{\boldsymbol{k}}\right\|_{p} \sum_{|\boldsymbol{k}|>n} C\|u\|_{W_{p}^{e}, \ell}\left\|\boldsymbol{h}_{\boldsymbol{k}}\right\|^{1+\ell / d} \\
& =C\|u\|_{W_{p}^{e, \ell}} \sum_{|\boldsymbol{k}|>n} 2^{-|\boldsymbol{k}|(1+\ell / d)} \\
& =C\|u\|_{W_{p}^{e, \ell}}\left(\begin{array}{c}
n+d \\
d-1
\end{array}\right) F\left(1,1+n+d ; 2+n ; 2^{-(1+\ell / d}\right) 2^{-n(1+\ell / d)}
\end{aligned}
$$

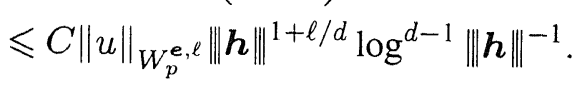

Theorem 3.7. Let $\widehat{R}_{n} u$ be the piecewise d-linear approximation of a function $u \in C_{0}^{e, 1}(\Omega)$ on a sparse grid on level $n$, as in Theorem 3.6, then, with $\|\boldsymbol{h}\|=2^{-n}$, the volume of the finest cells, we have, for $p=2, p=\infty$, the estimates

$$
\left\|u-\widehat{R}_{n} u\right\|_{W_{p}^{1}} \leqslant C\|\boldsymbol{h}\| \log ^{d-1}\|\boldsymbol{h}\|^{-1}\|u\|_{W_{p}^{e, 1}}
$$

If, moreover, we know $u \in C^{2 e}$, then

$$
\left\|u-\widehat{R}_{n} u\right\|_{W_{p}^{1}} \leqslant C\|\boldsymbol{h}\|\left\|D^{2 e} u\right\|_{p}
$$

Proof. Let $u$ be sufficiently differentiable and let $\mathbf{0} \leqslant \boldsymbol{m} \leqslant \boldsymbol{e}$ and $|\boldsymbol{m}| \geqslant 1$, then:

Part 1:

$$
\begin{aligned}
\left\|D^{\boldsymbol{m}} w_{\boldsymbol{k}}\right\|_{p} & =\left\|D^{\boldsymbol{m}} \prod_{j=1}^{d}\left(R_{\boldsymbol{k}}-R_{\boldsymbol{k}-\boldsymbol{e}_{j}}\right) u\right\|_{p} \\
& \leqslant C^{d}\left\|D^{\boldsymbol{m}} \prod_{j=1}^{d} h_{k_{j}} D^{\boldsymbol{e}_{j}} u\right\|_{p} \\
& \leqslant C^{d}\left\|\boldsymbol{h}_{\boldsymbol{k}}\right\|\left\|D^{\boldsymbol{m}} D^{\boldsymbol{e}} u\right\|_{p}
\end{aligned}
$$




$$
\begin{aligned}
\left\|D^{m}\left(u-\widehat{R}_{n} u\right)\right\|_{p} & \leqslant\left\|\sum_{|\boldsymbol{k}|>n} D^{m} w_{\boldsymbol{k}}\right\|_{p} \\
& \leqslant C^{d}\left\|D^{\boldsymbol{m}+\boldsymbol{e}} u\right\|_{p} \frac{n^{d-1} 2^{-n}}{(d-1) !} \bar{G}(n, d) \\
& \leqslant C\left\|D^{m+e} u\right\|_{p}\|\boldsymbol{h}\| \log ^{d-1}\|\boldsymbol{h}\| .
\end{aligned}
$$

Part 2:

$$
\begin{aligned}
\left\|D^{m}\left(u-\widehat{R}_{n} u\right)\right\|_{p} & \leqslant\left\|\sum_{|\boldsymbol{k}|>n} D^{m} w_{\boldsymbol{k}}\right\|_{p} \\
& \leqslant C\left\|D^{2 e} u\right\|_{p} 2^{-n}\left(C_{1} 2^{-n} n^{d-|m|-1}+C_{2} n^{|\boldsymbol{m}|-1}\right),
\end{aligned}
$$

with $C_{1}=0$ if $|\boldsymbol{m}|=d$, and $C_{2}=0$ if $|\boldsymbol{m}|=0$.

Because

$$
\|v\|_{\mathcal{W}_{p}^{1}}=\left(\|v\|_{p}^{p}+\sum_{|\boldsymbol{m}|=1,0 \leqslant \boldsymbol{m} \leqslant \boldsymbol{e}}\left\|D^{\boldsymbol{m}} v\right\|_{p}^{p}\right)^{1 / p}
$$

we consider the case $|\boldsymbol{m}|=1$ and we find

$$
\left\|u-\widehat{R}_{n} u\right\|_{W_{p}^{\prime}} \leqslant C\left\|D^{2 e} u\right\|_{p}\|\boldsymbol{h}\| .
$$

Together with the result of Theorem 3.6 this proves the theorem.

\section{References}

[1] G. Beylkin, Wavelets and Fast Numerical Algorithms, Proceedings of Symposia in Applied Mathematics 47 (American Mathematical Society, Providence, RI, 1993).

[2] H.J. Bungartz, Dünne Gitter und deren Anwendung bei der adaptiven Lösung der dreidimensionalen Poisson-Gleichung, Ph.D. Thesis, Institut für Informatik, TU München (1992).

[3] C.K. Chui, An Introduction to Wavelets (Academic Press, 1992).

[4] P.M. de Zeeuw, Development of semi-coarsening techniques, Applied Numerical Mathematics 19 (1996) $433-465$.

[5] J.E. Dendy, M.P. Ida and J.M. Rutledge, A semicoarsening multigrid algorithm for SIMD machines, SIAM J. Sci. Statist. Comput. 13 (1992) 1460-1469.

[6] M. Griebel, W. Huber, U. Rüde and T. Störtkuhl, The combination technique for parallel sparse-gridpreconditioning or -solution of PDEs on workstation networks, in: Parallel Processing: CONPAR 92VAPP V, Proceedings of the Second Joint International Conference on Vector and Parallel Processing, Lyon, France, September 1-3, 1992, Lecture Notes in Computer Science 634 (Springer, Berlin, 1992) 217-228.

[7] M. Griebel, M. Schneider and C. Zenger, A combination technique for the solution of sparse grid problems, in: Proceedings of the IMACS International Symposium on Iterative Methods in Linear Algebra (Elsevier, Amsterdam, 1992) 263-281.

[8] M. Griebel, C. Zenger and S. Zimmer, Multilevel Gauss-Seidel algorithms for full and sparse grid problems, Computing 50 (1993) 127-148.

[9] C.E. Heil and D.F Walnut, Continuous and discrete wavelet transforms, SIAM Rev. 31 (1989) 628-666. 
[10] P.W. Hemker, Sparse-grid finite-volume multigrid for 3D-problems, Adv. Comput. Math. 4 (1995) 83-110.

[11] P.W. Hemker and P.M. de Zeeuw, BASIS3: A data structure for 3-dimensional sparse grids, Technical Report NM-R9321, CWI, Amsterdam (1993).

[12] W.A. Mulder, A high resolution Euler solver based on multigrid, semi coarsening, and defect correction, J. Comput. Phys. 100 (1992) 91-104.

[13] C. Pflaum, Diskretisierung elliptischer Differentialgleichungen mit dünnen Gittern, Ph.D. Thesis, Technische Universität München, Munich (1995).

[14] R.A. Smith and A. Weiser, Semicoarsening multigrid on a hypercube, SIAM J. Sci. Statist. Comput. 13 (1992) 1314-1329.

[15] S.A. Smoljak, Quadrature and interpolation formulas for tensor product of certain classes of functions, Dokl. Akad. Nauk 148 (1963) 1042-1045; translation: Soviet Math. 4 (1963) 240-243.

[16] C. Zenger, Sparse grids, in: Parallel Algorithms for Partial Differential Equations: Proceedings of the Sixth GAMM-Seminar, Kiel, January 1990, Notes on Numerical Fluid Mechanics 31 (Vieweg, Braunschweig, 1991).

[17] L.-B. Zhang, Semi-coarsening in multigrid solution of steady incompressible Navier-Stokes equations, J. Comput. Math. 8 (1990) 92-97. 